DOI 10.4171/JEMS/223

Vincent Millot · Adriano Pisante

\title{
Symmetry of local minimizers for the three-dimensional Ginzburg-Landau functional
}

Received April 16, 2008 and in revised form September 29, 2008

\begin{abstract}
We classify nonconstant entire local minimizers of the standard Ginzburg-Landau functional for maps in $H_{\text {loc }}^{1}\left(\mathbb{R}^{3} ; \mathbb{R}^{3}\right)$ satisfying a natural energy bound. Up to translations and rotations, such solutions of the Ginzburg-Landau system are given by an explicit solution equivariant under the action of the orthogonal group.
\end{abstract}

Keywords. Ginzburg-Landau equation, harmonic maps, local minimizers

\section{Introduction}

Symmetry results for nonlinear elliptic PDE's are difficult and usually rely on a clever use of the maximum principle as in the celebrated Serrin's moving planes method, or the use of rearrangement techniques as the Schwarz symmetrization (see, e.g., [7] and [19] for a survey). In the case of systems the situation is more involved since there are no general tools for proving this kind of results.

In this paper we investigate symmetry properties of maps $u: \mathbb{R}^{3} \rightarrow \mathbb{R}^{3}$ which are entire (smooth) solutions of the system

$$
\Delta u+u\left(1-|u|^{2}\right)=0
$$

possibly subject to the condition at infinity

$$
|u(x)| \rightarrow 1 \quad \text { as }|x| \rightarrow+\infty .
$$

The system 1.1 is naturally associated to the energy functional

$$
E(v, \Omega):=\int_{\Omega}\left(\frac{1}{2}|\nabla v|^{2}+\frac{1}{4}\left(1-|v|^{2}\right)^{2}\right) d x
$$

defined for $v \in H_{\text {loc }}^{1}\left(\mathbb{R}^{3} ; \mathbb{R}^{3}\right)$ and a bounded open set $\Omega \subset \mathbb{R}^{3}$. Indeed, if $u \in H_{\text {loc }}^{1}\left(\mathbb{R}^{3} ; \mathbb{R}^{3}\right)$ is a critical point of $E(\cdot, \Omega)$ for every $\Omega$ then $u$ is a weak solution of $(1.1)$ and thus

V. Millot: Université Paris Diderot - Paris 7, CNRS, UMR 7598 Laboratoire Jacques-Louis Lions, F-75005 Paris, France; e-mail: millot@math.jussieu.fr

A. Pisante: Department of Mathematics, University of Rome 'La Sapienza', P.le Aldo Moro 5, 00185 Roma, Italy; e-mail: pisante@mat.uniroma1.it

Mathematics Subject Classification (2010): 35J50, 58E20, 58J70 
a classical solution according to the standard regularity theory for elliptic equations. In addition, any weak solution $u$ of (1.1) satisfies the natural bound $|u| \leq 1$ in the entire space (see [12, Proposition 1.9]).

Here the "boundary condition" $(1.2$ ) is added to rule out solutions with values in a lower dimensional Euclidean space like the scalar valued solutions relevant for the De Giorgi conjecture (see, e.g., [3]), or the explicit vortex solutions of [17] (see also [16] and [6]) arising in the 2D Ginzburg-Landau model. More precisely, under the assumption 1.2. the map $u$ has a well defined topological degree at infinity given by

$$
\operatorname{deg}_{\infty} u:=\operatorname{deg}\left(\frac{u}{|u|}, \partial B_{R}\right)
$$

whenever $R$ is large enough, and we are interested in solutions satisfying $\operatorname{deg}_{\infty} u \neq 0$. A special symmetric solution $U$ to $1.1-1.2$ with $\operatorname{deg}_{\infty} U=1$ has been constructed in [1] and [15] in the form

$$
U(x)=\frac{x}{|x|} f(|x|),
$$

for a unique function $f$ vanishing at zero and increasing to one at infinity. Taking into account the obvious invariance properties of (1.1) and (1.3), infinitely many solutions can be obtained from (1.4) by translations on the domain and orthogonal transformations on the image. In addition, these solutions satisfy $R^{-1} E\left(u, B_{R}\right) \rightarrow 4 \pi$ as $R \rightarrow+\infty$. It is easy to check that $U$ as in (1.4) is the unique solution $u$ of $(1.1)-(1.2)$ such that $u^{-1}(\{0\})=\{0\}, \operatorname{deg}_{\infty} u=1$ and $u$ is $O(3)$-equivariant, i.e., $u(T x)=T u(x)$ for all $x \in \mathbb{R}^{3}$ and all $T \in O(3)$ (see Remark 2.1). In addition $u=U$ satisfies $|u(x)|=$ $1+\mathcal{O}\left(|x|^{-2}\right)$ as $|x| \rightarrow+\infty$.

In [7], H. Brezis has formulated the following problem:

Is any solution to (1.1) satisfying (1.2) (possibly with a "good" rate of convergence) and $\operatorname{deg}_{\infty} u= \pm 1$, of the form (1.4) (up to a translation on the domain and an orthogonal transformation on the image)?

In this paper we investigate this problem focusing on local minimizers of the energy in the following sense.

Definition 1.1. Let $u \in H_{\mathrm{loc}}^{1}\left(\mathbb{R}^{3} ; \mathbb{R}^{3}\right)$. We say that $u$ is a local minimizer of $E(\cdot)$ if

$$
E(u, \Omega) \leq E(v, \Omega)
$$

for any bounded open set $\Omega \subset \mathbb{R}^{3}$ and $v \in H_{\text {loc }}^{1}\left(\mathbb{R}^{3} ; \mathbb{R}^{3}\right)$ satisfying $v-u \in H_{0}^{1}\left(\Omega ; \mathbb{R}^{3}\right)$.

Obviously local minimizers are smooth entire solutions of (1.1) but it is not clear that nonconstant local minimizers do exist or if the solutions obtained from (1.4) are locally minimizing. In the case of maps from the plane into itself the analogous problems are of importance in the study of the asymptotic behavior of minimizers of the $2 \mathrm{D}$ Ginzburg-Landau energy near their vortices, the explicit solutions of the form 1.4 giving the asymptotic profile of the minimizers in the vortex cores. Both these questions were essentially solved affirmatively in [23, 24, 26] (see also [25] for the more difficult 
gauge-dependent problem, i.e., in the presence of a magnetic field) but the complete classification of entire solutions to (1.1)-(1.2), even in the 2D case, remains open.

The first result of this paper concerns the existence of nonconstant local minimizers.

Theorem 1.1. There exists a smooth nonconstant solution $u: \mathbb{R}^{3} \rightarrow \mathbb{R}^{3}$ of 1.1 - 1.2 which is a local minimizer of $E(\cdot)$. In addition, $u(0)=0, \operatorname{deg}_{\infty} u=1$ and $R^{-1} E\left(u, B_{R}\right)$ $\rightarrow 4 \pi$ as $R \rightarrow+\infty$.

The construction of a nonconstant local minimizer relies on a careful analysis of the vorticity set for solutions $u_{\lambda}$ to

$$
\left(P_{\lambda}\right)\left\{\begin{array}{ll}
\Delta u+\lambda^{2} u\left(1-|u|^{2}\right)=0 & \text { in } B_{1}, \\
u=\mathrm{Id} & \text { on } \partial B_{1},
\end{array} \quad \lambda>0,\right.
$$

which are absolute minimizers of the Ginzburg-Landau functional $E_{\lambda}\left(u, B_{1}\right)$ on $H_{\mathrm{Id}}^{1}\left(B_{1} ; \mathbb{R}^{3}\right)$ where

$$
E_{\lambda}(u, \Omega):=\int_{\Omega} e_{\lambda}(u) d x \quad \text { with } \quad e_{\lambda}(u):=\frac{1}{2}|\nabla u|^{2}+\frac{\lambda^{2}}{4}\left(1-|u|^{2}\right)^{2} .
$$

Up to a translation, we will obtain a locally minimizing solution to 1.1 as a limit of $u_{\lambda_{n}}\left(x / \lambda_{n}\right)$ for some sequence $\lambda_{n} \rightarrow+\infty$.

As the smooth entire solutions of (1.1), critical points of the energy functional $E_{\lambda}(\cdot, \Omega)$ satisfy a fundamental monotonicity identity (see [27], [22]).

Lemma 1.1 (Monotonicity Formula). Assume that $u: \Omega \rightarrow \mathbb{R}^{3}$ solves $\Delta u+\lambda^{2} u\left(1-|u|^{2}\right)$ $=0$ in some open set $\Omega \subset \mathbb{R}^{3}$ and $\lambda>0$. Then

$$
\begin{aligned}
\frac{1}{R} E_{\lambda}\left(u, B_{R}\left(x_{0}\right)\right)= & \frac{1}{r} E_{\lambda}\left(u, B_{r}\left(x_{0}\right)\right)+\int_{B_{R}\left(x_{0}\right) \backslash B_{r}\left(x_{0}\right)} \frac{1}{\left|x-x_{0}\right|}\left|\frac{\partial u}{\partial\left|x-x_{0}\right|}\right|^{2} d x \\
& +\frac{\lambda^{2}}{2} \int_{r}^{R} \frac{1}{t^{2}} \int_{B_{t}\left(x_{0}\right)}\left(1-|u|^{2}\right)^{2} d x d t,
\end{aligned}
$$

for any $x_{0} \in \Omega$ and any $0<r \leq R \leq \operatorname{dist}\left(x_{0}, \partial \Omega\right)$.

An entire solution $u$ to 1.1 ) for which the left hand side of 1.7 ( with $\lambda=1$ ) is bounded, i.e.,

$$
\sup _{R>0} R^{-1} E\left(u, B_{R}\right)<+\infty,
$$

can be studied near infinity through a "blow-down" analysis. More precisely, for each $R>0$ we introduce the scaled map $u_{R}$ defined by

$$
u_{R}(x):=u(R x),
$$

which is a smooth entire solution of

$$
\Delta u_{R}+R^{2} u_{R}\left(1-\left|u_{R}\right|^{2}\right)=0 .
$$


Whenever $E\left(u, B_{R}\right)$ grows at most linearly with $R, E_{R}\left(u_{R}, \Omega\right)$ is equibounded and thus $\left\{u_{R}\right\}_{R>0}$ is bounded in $H_{\mathrm{loc}}^{1}\left(\mathbb{R}^{3} ; \mathbb{R}^{3}\right)$. Any weak limit $u_{\infty}: \mathbb{R}^{3} \rightarrow \mathbb{R}^{3}$ of $\left\{u_{R}\right\}_{R>0}$ as $R \rightarrow+\infty$ is called a tangent map to $u$ at infinity, and the potential term in the energy forces $u_{\infty}$ to take values in $\mathbb{S}^{2}$. Moreover (see [22]), $u_{\infty}$ turns out to be harmonic and positively 0-homogeneous, i.e., $u_{\infty}(x)=\omega(x /|x|)$ for some harmonic map $\omega: \mathbb{S}^{2} \rightarrow \mathbb{S}^{2}$, and $u_{\infty}$ is a solution or a critical point (among $\mathbb{S}^{2}$-valued maps) of

$$
\Delta v+v|\nabla v|^{2}=0, \quad E_{\infty}(v, \Omega)=\int_{\Omega} \frac{1}{2}|\nabla v|^{2} d x,
$$

respectively. This is readily the case for the equivariant solution (1.4), where $U_{R}(x) \rightarrow$ $x /|x|$ strongly in $H_{\text {loc }}^{1}\left(\mathbb{R}^{3} ; \mathbb{R}^{3}\right)$ as $R \rightarrow+\infty$. In the general case, uniqueness of the tangent map at infinity is not guaranteed and the possible lack of compactness of $\left\{u_{R}\right\}_{R>0}$ has been carefully analyzed in [21, 22] where the blow-up analysis of the defect measure arising in the limit of the measures $e_{R}\left(u_{R}\right) d x$ is performed. As a byproduct (see [22, Corollary D]), a quantization result for the normalized energy is obtained, namely $R^{-1} E\left(u, B_{R}\right) \rightarrow 4 \pi k$ as $R \rightarrow+\infty$ for some $k \in \mathbb{N}$, the case $k=1$ being valid both for the solution (1.4) (see Proposition 2.1) and the local minimizer constructed in Theorem 1.1 The following result shows that the same property is true for any local minimizer of $E(\cdot)$ satisfying $(1.8$, so that any nonconstant local minimizer of $E(\cdot)$ satisfying 1.8 ) realizes the lowest energy quantization level.

Theorem 1.2. Let $u \in H_{\mathrm{loc}}^{1}\left(\mathbb{R}^{3} ; \mathbb{R}^{3}\right)$ be a nonconstant local minimizer of $E(\cdot)$ satisfying 1.8. Then $R^{-1} E\left(u, B_{R}\right) \rightarrow 4 \pi$ as $R \rightarrow+\infty$ and the scaled maps $\left\{u_{R}\right\}_{R>0}$ are relatively compact in $H_{\mathrm{loc}}^{1}\left(\mathbb{R}^{3} ; \mathbb{R}^{3}\right)$.

In proving this theorem, the first step is to apply the blow-down analysis from infinity given in [22]. Then, taking minimality into account, we exclude concentration by a comparison argument involving a "dipole removing technique". This yields the compactness of the scaled maps. Finally another comparison argument based on minimality and on the results in [8] gives the desired value for the limit of the scaled energy. Here we believe that (as shown in [26] for the 2D case) assumption (1.8) should always hold, as a consequence of local minimality.

In order to prove full symmetry of a nonconstant local minimizer, a natural approach is to prove uniqueness and symmetry of the tangent map at infinity, and then try to propagate the symmetry from infinity to the entire space. As a first step in this direction, we have the following result inspired by the asymptotic analysis developed for harmonic maps at isolated singularities in the important work [29] (see also [30], [18] for a possibly simplified treatment and a more comprehensive exposition of the subject, and [14] for the case of $\mathbb{S}^{2}$-valued harmonic maps in $\mathbb{R}^{3}$ ).

Theorem 1.3. Let $u$ be an entire smooth solution of (1.1) satisfying $(1.8)$ and such that the scaled maps $\left\{u_{R}\right\}_{R>0}$ are relatively compact in $H_{\mathrm{loc}}^{1}\left(\mathbb{R}^{3} ; \mathbb{R}^{3}\right)$. Then there exist a constant $C>0$ such that for all $x \in \mathbb{R}^{3}$,

$$
|x|^{2}\left(1-|u(x)|^{2}\right)+|x||\nabla u(x)|+|x|^{3}\left|\nabla\left(1-|u(x)|^{2}\right)\right|+|x|^{2}\left|\nabla^{2} u(x)\right| \leq C,
$$


and there exists a unique harmonic map $\omega: \mathbb{S}^{2} \rightarrow \mathbb{S}^{2}$ such that $\operatorname{deg} \omega=\operatorname{deg}_{\infty} u$ and setting $u_{\infty}(x)=\omega(x /|x|)$,

(i) $\left\|u_{R \mid \mathbb{S}^{2}}-\omega\right\|_{C^{2}\left(\mathbb{S}^{2} ; \mathbb{R}^{3}\right)} \rightarrow 0$ as $R \rightarrow+\infty$,

(ii) $e_{R}\left(u_{R}\right)(x) d x \stackrel{*}{\rightarrow} \frac{1}{2}\left|\nabla u_{\infty}\right|^{2} d x$ weakly* as measures as $R \rightarrow+\infty$.

If in addition $\operatorname{deg}_{\infty} u= \pm 1$ then $\omega(x)=T x$ for some $T \in O(3)$.

This result strongly relies on the a priori bound (1.11) for entire solutions to (1.1) which, loosely speaking, do not exibit any bubbling phenomena at infinity (more precisely, the scaled maps $\left\{u_{R}\right\}$ do not exibit energy concentration as $R \rightarrow+\infty$ ). Whenever (1.11) holds, we can write for $|x|$ sufficiently large the polar decomposition of the solution $u$ as $u(x)=\rho(x) w(x)$ for some positive function $\rho$ and some $\mathbb{S}^{2}$-valued map $w$ which have to solve the system

$$
\left\{\begin{array}{l}
\operatorname{div}\left(\rho^{2}(x) \nabla w(x)\right)+w(x) \rho^{2}(x)|\nabla w(x)|^{2}=0, \\
\Delta \rho(x)+\rho(x)\left(1-\rho^{2}(x)\right)=\rho(x)|\nabla w(x)|^{2}
\end{array}\right.
$$

for $|x|$ large. It is clear from (1.11) that $\rho$ smoothly tends to 1 at infinity. Hence the unit map $w$ tends to be harmonic as $|x| \rightarrow+\infty$, and system (1.12) can be considered as a perturbation of the harmonic map system. In the present situation, uniqueness of the asymptotic limit can be obtained from an elementary but tricky estimate on the radial derivative of $w$, and we avoid the use of the Simon-Łojasiewicz inequality.

Once the asymptotic symmetry is obtained we can adapt the division method used in [24] and [25] to get full symmetry. The main result of the paper is the following.

Theorem 1.4. Let $u$ be an entire solution of (1.1). The following conditions are equivalent:

(i) $u$ is a nonconstant local minimizer of $E(\cdot)$ satisfying [1.8];

(ii) $E\left(u, B_{R}\right)=4 \pi R+o(R)$ as $R \rightarrow+\infty$;

(iii) $|u(x)|=1+\mathcal{O}\left(|x|^{-2}\right)$ as $|x| \rightarrow+\infty$ and $\operatorname{deg}_{\infty} u= \pm 1$;

(iv) up to a translation on the domain and an orthogonal transformation on the image, $u$ is $O$ (3)-equivariant, i.e., $u=U$ as given by 1.4 .

As a consequence of this theorem, we see that under the assumption (1.8), up to translations and orthogonal transformations, any nonconstant local minimizer of $E_{\lambda}(\cdot)$ in $H_{\text {loc }}^{1}\left(\mathbb{R}^{3} ; \mathbb{R}^{3}\right)$ is given by $u(x)=U(\lambda x)$ with $U$ as in 1.4 . In the limiting case $\lambda=+\infty$, a similar result has been proved in [2, Theorem 2.2] showing that any nonconstant local minimizer $u$ of the Dirichlet integral $E_{\infty}(\cdot)$ in $H_{\text {loc }}^{1}\left(\mathbb{R}^{3} ; \mathbb{S}^{2}\right)$ is given by $u(x)=x /|x|$ up to translations and orthogonal transformations.

The plan of the paper is the following. In Section 2 we review the properties of the equivariant solution (1.47. In Section 3 we study minimizing solutions to $\left(P_{\lambda}\right)$ and prove Theorem 1.1. In Section 4 we prove the quantization property for an arbitrary local minimizer, i.e., we prove Theorem 1.2 In Section 5 we deal with asymptotic symmetry and Theorem 1.3 . Finally in Section 6 we obtain the full symmetry and the main result of the paper. 


\section{The equivariant solution}

In this section we collect some preliminary results about equivariant entire solutions. The existence statement and the qualitative study are essentially contained in [1, 13] and [15]. In the following lemma we stress the asymptotic decay at infinity.

Lemma 2.1. There is a unique solution $f \in C^{2}([0,+\infty))$ of

$$
\left\{\begin{array}{l}
f^{\prime \prime}+\frac{2}{r} f^{\prime}-\frac{2}{r^{2}} f+f\left(1-f^{2}\right)=0 \\
f(0)=0 \text { and } f(+\infty)=1
\end{array}\right.
$$

In addition $0<f(r)<1$ for each $r>0, f^{\prime}(0)>0, f$ is strictly increasing,

$$
R^{2}\left|f^{\prime \prime}(R)\right|+R f^{\prime}(R)+\left|2-R^{2}\left(1-f(R)^{2}\right)\right|=o(1) \quad \text { as } R \rightarrow+\infty,
$$

and

$$
\frac{1}{R} \int_{0}^{R}\left(\frac{r^{2}}{2}\left(f^{\prime}\right)^{2}+f^{2}+r^{2} \frac{\left(1-f^{2}\right)^{2}}{4}\right) d r \rightarrow 1 \quad \text { as } R \rightarrow+\infty .
$$

Proof. The existence of an increasing solution follows from [15] and [1]. To obtain the estimates at infinity in 2.2), we multiply the equation by $r^{2} f^{\prime}(r)$ and an integration by parts yields

$$
\frac{R^{2}}{2}\left(f^{\prime}(R)\right)^{2}+\int_{0}^{R} r\left(f^{\prime}(r)\right)^{2} d r+\int_{0}^{R} r^{2}\left(1-(f(r))^{2}\right) f(r) f^{\prime}(r) d r=(f(R))^{2} \leq 1 .
$$

Using the monotonicity of $f$, we deduce that $\int_{0}^{+\infty} r\left(f^{\prime}(r)\right)^{2} d r<+\infty$. Hence we can find a sequence $R_{n} \rightarrow+\infty$ such that $R_{n} f^{\prime}\left(R_{n}\right) \rightarrow 0$ as $n \rightarrow+\infty$. On the other hand the integral terms in 2.4 admit a limit as $R \rightarrow+\infty$. As a consequence, $r f^{\prime}(r)$ admits a limit at infinity and thus $R f^{\prime}(R) \rightarrow 0$ as $R \rightarrow+\infty$. For any $k \in(0,1)$ fixed, multiplying the equation by $r^{2}$ and averaging over $(k R, R)$ leads to

$$
\begin{aligned}
\frac{R^{2} f^{\prime}(R)-k^{2} R^{2} f^{\prime}(k R)}{(1-k) R}+\frac{1}{(1-k) R} \int_{k R}^{R} f(r) r^{2}(1- & \left.(f(r))^{2}\right) d r \\
& =\frac{2}{(1-k) R} \int_{k R}^{R} f(r) d r .
\end{aligned}
$$

Since $f$ is increasing and tends to 1 at infinity, we infer

$$
k^{2} \limsup _{R \rightarrow+\infty} R^{2}\left(1-(f(R))^{2}\right) \leq 2 \leq \liminf _{R \rightarrow+\infty} R^{2}\left(1-(f(k R))^{2}\right),
$$

so that $R^{2}\left(1-(f(R))^{2}\right) \rightarrow 2$ as $R \rightarrow+\infty$ by arbitrariness of $k$. Taking the equation into account, 2.2 follows. To prove 2.3 we multiply the equation by $r^{2}\left(1-f^{2}\right)$ and we integrate by parts on $(0, R)$ to get

$$
R^{2}\left(1-(f(R))^{2}\right) f^{\prime}(R)+2 \int_{0}^{R} r^{2} f\left(f^{\prime}\right)^{2} d r+\int_{0}^{R} r^{2} f\left(1-f^{2}\right)^{2} d r=2 \int_{0}^{R} f\left(1-f^{2}\right) d r .
$$


Since $f$ is increasing and tends to 1 at infinity, we deduce using 2.2 that

$$
\frac{1}{R} \int_{0}^{R} r^{2}\left(1-f^{2}\right)^{2} d r+\frac{1}{R} \int_{0}^{R} 2 r^{2}\left(f^{\prime}\right)^{2} d r+R^{2}\left(1-(f(R))^{2}\right) f^{\prime}(R) \rightarrow 0,
$$

and 2.3 follows easily.

A consequence of the previous lemma is the following result.

Proposition 2.1. Let $x_{0} \in \mathbb{R}^{3}$ and $T \in O(3)$. Consider the function $f:[0,+\infty) \rightarrow$ $[0,1)$ given by Lemma 2.1 and define

$$
w(x):=\frac{T\left(x-x_{0}\right)}{\left|x-x_{0}\right|} f\left(\left|x-x_{0}\right|\right) .
$$

Then $w$ is a smooth solution of (1.1). In addition, $0<|w(x)|<1$ for each $x \neq x_{0}$, $w$ satisfies 1.11, and

$$
\lim _{R \rightarrow+\infty} \frac{1}{R} \int_{B_{R}\left(x_{0}\right)}\left(\frac{1}{2}|\nabla w(x)|^{2}+\frac{\left(1-|w(x)|^{2}\right)^{2}}{4}\right) d x=4 \pi .
$$

Proof. As in [1] and [15], $w$ is smooth and it is a classical solution of [1.1]. It is routine to check that 1.11 follows from 2.2 . Then a simple calculation yields

$$
|\nabla w(x)|^{2}=\left(f^{\prime}\left(\left|x-x_{0}\right|\right)\right)^{2}+\frac{2\left(f\left(\left|x-x_{0}\right|\right)\right)^{2}}{\left|x-x_{0}\right|^{2}}+\frac{\left(1-\left|f\left(\left|x-x_{0}\right|\right)\right|^{2}\right)^{2}}{4},
$$

whence 2.5 follows from 2.3.

Remark 2.1. The solution $U$ given by (1.4) is the unique $O(3)$-equivariant solution $u$ of 1.1 -1.2 such that $u^{-1}(\{0\})=\{0\}$ and $\operatorname{deg}_{\infty} u=1$. Indeed, for each fixed $x \neq 0$, setting $l_{x}$ to be the line passing through 0 and $x, u\left(l_{x}\right) \subset l_{x}$ because $u$ is equivariant (actually invariant) under rotations fixing $l_{x}$. Hence we can write $u(x)=(x /|x|) \sigma(x)|u(x)|$ with $\sigma(x)= \pm 1$ and $|u(x)|=g(|x|)$ for some smooth function $g:(0,+\infty) \rightarrow(0,+\infty)$. Since $u$ is smooth and $\operatorname{deg}_{\infty} u=1$, we conclude that $\sigma \equiv 1$. Taking (1.2) into account we conclude that $g$ satisfies the Cauchy problem 2.1). Finally by the uniqueness result in [1, 15], we obtain $g \equiv f$ as claimed.

\section{Existence of nonconstant local minimizers}

A basic ingredient in the construction of a nonconstant local minimizer is the following small energy regularity result taken from [22] (see also [11]).

Lemma 3.1. There exist positive constants $\eta_{0}>0$ and $C_{0}>0$ such that for any $\lambda \geq 1$ and any $u \in C^{2}\left(B_{2 R}\left(x_{0}\right) ; \mathbb{R}^{3}\right)$ satisfying

$$
\Delta u+\lambda^{2} u\left(1-|u|^{2}\right)=0 \quad \text { in } B_{2 R}\left(x_{0}\right),
$$

with $\frac{1}{2 R} E_{\lambda}\left(u, B_{2 R}\left(x_{0}\right)\right) \leq \eta_{0}$, we have

$$
R^{2} \sup _{B_{R}\left(x_{0}\right)} e_{\lambda}(u) \leq C_{0} \frac{1}{2 R} E_{\lambda}\left(u, B_{2 R}\left(x_{0}\right)\right) .
$$

We will also make use of the following boundary version of Lemma 3.1 (see [9, 10]). 
Lemma 3.2. Let $g: \partial B_{1} \rightarrow \mathbb{S}^{2}$ be a smooth map. There exist positive constants $\eta_{1}>0$ and $C_{1}>0$ such that for any $\lambda \geq 1,0<R<\eta_{1} / 2, x_{0} \in \partial B_{1}$ and any $u \in$ $C^{2}\left(\bar{B}_{1} \cap B_{2 R}\left(x_{0}\right) ; \mathbb{R}^{3}\right)$ satisfying $u=g$ on $\partial B_{1} \cap B_{2 R}\left(x_{0}\right)$ and

$$
\Delta u+\lambda^{2} u\left(1-|u|^{2}\right)=0 \quad \text { in } B_{1} \cap B_{2 R}\left(x_{0}\right),
$$

with $\frac{1}{2 R} E_{\lambda}\left(u, B_{1} \cap B_{2 R}\left(x_{0}\right)\right) \leq \eta_{1}$, we have

$$
R^{2} \sup _{B_{1} \cap B_{R}\left(x_{0}\right)} e_{\lambda}(u) \leq C_{1} \frac{1}{2 R} E_{\lambda}\left(u, B_{1} \cap B_{2 R}\left(x_{0}\right)\right) .
$$

Another result which is a combination of [21] and [22] will play a crucial role.

Proposition 3.1. Let $\Omega \subset \mathbb{R}^{3}$ be a smooth bounded open set and let $\lambda_{n} \rightarrow+\infty$ as $n \rightarrow+\infty$. For every $n \in \mathbb{N}$ let $u_{n}$ be a critical point of $E_{\lambda_{n}}(\cdot, \Omega)$ such that $\sup _{n} E_{\lambda_{n}}\left(u_{n}, \Omega\right)<+\infty$. Then, up to a subsequence, $u_{n} \rightarrow u$ weakly in $H^{1}\left(\Omega ; \mathbb{R}^{3}\right)$ for some weakly harmonic map $u: \Omega \rightarrow \mathbb{S}^{2}$, and $e_{\lambda_{n}}\left(u_{n}\right)(x) d x \stackrel{*}{\rightarrow} \frac{1}{2}|\nabla u|^{2} d x+v$ weakly* as measures on $\Omega$ where $v=4 \pi \theta \mathcal{H}^{1}\left\llcorner\Sigma\right.$ for some $\mathcal{H}^{1}$-rectifiable set $\Sigma$ of locally finite $\mathcal{H}^{1}$-measure and some integer valued $\mathcal{H}^{1}$-measurable function $\theta: \Sigma \rightarrow \mathbb{N}$.

The key result of this section is the following proposition.

Proposition 3.2. Let $\lambda \geq 1$ and $u_{\lambda} \in H^{1}\left(B_{1} ; \mathbb{R}^{3}\right)$ be a global minimizer of $E_{\lambda}\left(\cdot, B_{1}\right)$ over $H_{\mathrm{Id}}^{1}\left(B_{1} ; \mathbb{R}^{3}\right)$. For any $\delta \in(0,1)$, there exists a constant $C_{\delta}>0$ independent of $\lambda$ such that $\operatorname{diam}\left(\left\{\left|u_{\lambda}\right| \leq \delta\right\}\right) \leq C_{\delta} \lambda^{-1}$ and $\operatorname{dist}_{H}\left(\left\{\left|u_{\lambda}\right| \leq \delta\right\},\{0\}\right)=o(1)$ as $\lambda \rightarrow+\infty$ where dist $_{H}$ denotes the Hausdorff distance.

Proof. Let us consider an arbitrary sequence $\lambda_{n} \rightarrow+\infty$, and for every $n \in \mathbb{N}$ let $u_{n} \in H^{1}\left(B_{1} ; \mathbb{R}^{3}\right)$ be a global minimizer of $E_{\lambda_{n}}\left(\cdot, B_{1}\right)$ under the boundary condition $u_{n \mid \partial B_{1}}=x$. It is well known that $u_{n}$ satisfies $u_{n} \in C^{2}\left(\bar{B}_{1}\right)$ and $\left|u_{n}\right| \leq 1$ for every $n \in \mathbb{N}$.

Step 1 . We claim that $u_{n} \rightarrow v(x):=x /|x|$ strongly in $H^{1}\left(B ; \mathbb{R}^{3}\right)$. Since the map $v$ is admissible, one has

$$
\begin{aligned}
\frac{1}{2} \int_{B_{1}}\left|\nabla u_{n}\right|^{2} & \leq E_{\lambda_{n}}\left(u_{n}, B_{1}\right) \leq E_{\lambda_{n}}\left(v, B_{1}\right)=\frac{1}{2} \int_{B_{1}}|\nabla v|^{2} \\
& =4 \pi \quad \text { for every } n \in \mathbb{N} .
\end{aligned}
$$

As a consequence, $\left\{u_{n}\right\}$ is bounded in $H^{1}\left(B_{1} ; \mathbb{R}^{3}\right)$, and up to a subsequence, $u_{n} \rightarrow u_{\star}$ weakly in $H^{1}\left(B ; \mathbb{R}^{3}\right)$ for some $\mathbb{S}^{2}$-valued map $u_{\star}$ satisfying $u_{\star \mid \partial B_{1}}=x$. By Theorem 7.1 in [8], the map $v$ is the unique minimizer of $u \in H^{1}\left(B_{1} ; \mathbb{S}^{2}\right) \mapsto \int_{B_{1}}|\nabla u|^{2}$ under the boundary condition $u_{\mid \partial B_{1}}=x$. In particular, $\int_{B_{1}}\left|\nabla u_{\star}\right|^{2} \geq \int_{B_{1}}|\nabla v|^{2}$, which, combined with 3.3, yields

$$
\frac{1}{2} \int_{B_{1}}\left|\nabla u_{n}\right|^{2} \rightarrow \frac{1}{2} \int_{B_{1}}\left|\nabla u_{\star}\right|^{2}=\frac{1}{2} \int_{B_{1}}|\nabla v|^{2} \quad \text { as } n \rightarrow+\infty .
$$

Therefore $u_{\star} \equiv v$ and $u_{n} \rightarrow v$ strongly in $H^{1}\left(B ; \mathbb{R}^{3}\right)$. 
Step 2. Let $\delta \in(0,1)$ be fixed. We now prove that the compact sets $\mathcal{V}_{n}:=\left\{\left|u_{n}\right| \leq \delta\right\}$ tend to $\{0\}$ in the Hausdorff sense. It suffices to prove that for any given $0<\rho<1$, $\mathcal{V}_{n} \subset B_{\rho}$ for every $n$ large enough. Since $v$ is smooth outside the origin, we can find $0<\sigma \leq \min \left(\rho / 8, \eta_{1} / 4\right)$ such that

$$
\frac{1}{\sigma} \int_{B_{1} \cap B_{4 \sigma}(x)}|\nabla v|^{2}<\min \left(\eta_{0}, \eta_{1}\right)=: \ell \quad \text { for every } x \in \bar{B}_{1} \backslash B_{\rho},
$$

where $\eta_{0}$ and $\eta_{1}$ are given by Lemmas 3.1 and 3.2 respectively. From the strong convergence of $u_{n}$ to $v$ in $H^{1}$, we infer that

$$
\frac{1}{\sigma} E_{\lambda_{n}}\left(u_{n}, B_{4 \sigma}(x)\right)<\ell \quad \text { for every } x \in \bar{B}_{1} \backslash B_{\rho}
$$

whenever $n \geq N_{1}$, for some integer $N_{1}$ independent of $x$. Next consider a finite family of points $\left\{x_{j}\right\}_{j \in J} \subset \bar{B}_{1} \backslash B_{\rho}$ satisfying $B_{2 \sigma}\left(x_{j}\right) \subset B_{1}$ if $x_{j} \in B_{1}$ and

$$
\bar{B}_{1} \backslash B_{\rho} \subset\left(\bigcup_{x_{j} \in B_{1}} B_{\sigma}\left(x_{j}\right)\right) \cup\left(\bigcup_{x_{j} \in \partial B_{1}} B_{2 \sigma}\left(x_{j}\right)\right) .
$$

In view of (3.4), for each $j \in J$ we can apply Lemma 3.1 in $B_{2 \sigma}\left(x_{j}\right)$ if $x_{j} \in B_{1}$ and Lemma 3.2 in $B_{1} \cap B_{4 \sigma}\left(x_{j}\right)$ if $x_{j} \in \partial B_{1}$ to deduce

$$
\sup _{\bar{B}_{1} \backslash B_{\rho}} e_{\lambda_{n}}\left(u_{n}\right) \leq C \sigma^{-2} \quad \text { for every } n \geq N_{1},
$$

for some constant $C$ independent of $n$. By the Ascoli Theorem the sequence $\left\{u_{n}\right\}$ is compact in $C^{0}\left(\overline{B_{1}} \backslash B_{\rho}\right)$, and thus $\left|u_{n}\right| \rightarrow 1$ uniformly in $\overline{B_{1}} \backslash B_{\rho}$. In particular $\left|u_{n}\right|>\delta$ in $\overline{B_{1}} \backslash B_{\rho}$ whenever $n$ is large enough.

In the remainder of this proof we will establish the estimate $\operatorname{diam}\left(\mathcal{V}_{n}\right) \leq C_{\delta} \lambda_{n}^{-1}$. We shall argue by contradiction. Setting $r_{n}:=\operatorname{diam}\left(\mathcal{V}_{n}\right)$, we assume that for a subsequence, $\kappa_{n}:=r_{n} \lambda_{n} \rightarrow+\infty$. Let $a_{n}, b_{n} \in \mathcal{V}_{n}$ be such that $\left|a_{n}-b_{n}\right|=r_{n}$ and set $c_{n}$ to be the middle point of the segment $\left[a_{n}, b_{n}\right]$. In view of Step 2 , we have $c_{n} \rightarrow 0$. Next we define, for $n$ large enough and $x \in B_{2}$,

$$
w_{n}(x):=u_{n}\left(r_{n} x+c_{n}\right),
$$

so that $w_{n}$ satisfies

$$
\Delta w_{n}+\kappa_{n}^{2} w_{n}\left(1-\left|w_{n}\right|^{2}\right)=0 \quad \text { in } B_{2} .
$$

Up to a rotation, we may assume without loss of generality that $\left(a_{n}-c_{n}\right) / r_{n}=(1 / 2,0,0)$ $=: P_{1}$ and $\left(b_{n}-c_{n}\right) / r_{n}=(-1 / 2,0,0)=: P_{2}$ so that

$$
\left|w_{n}\left(P_{1}\right)\right|=\left|w_{n}\left(P_{2}\right)\right|=\delta \quad \text { for every } n \text { sufficiently large. }
$$

Step 3. We claim that up to a subsequence, $w_{n} \rightarrow \phi$ strongly in $H_{\text {loc }}^{1}\left(B_{2} ; \mathbb{R}^{3}\right)$ for some weakly stationary harmonic map $\phi: B_{2} \rightarrow \mathbb{S}^{2}$. First we infer from (3.3) and the Mono- 
tonicity Formula (1.7) applied to $w_{n}$ and $u_{n}$ that

$$
\begin{aligned}
\frac{1}{R} E_{\kappa_{n}}\left(w_{n}, B_{R}\left(x_{0}\right)\right) & \leq \frac{1}{1-\left|r_{n} x_{0}+c_{n}\right|} E_{\lambda_{n}}\left(u_{n}, B_{1-\left|r_{n} x_{0}+c_{n}\right|}\left(r_{n} x_{0}+c_{n}\right)\right) \\
& \leq \frac{4 \pi}{1-\left|r_{n} x_{0}+c_{n}\right|}
\end{aligned}
$$

for every $x_{0} \in B_{2}$ and $0<R<\operatorname{dist}\left(x_{0}, \partial B_{2}\right)$. Hence $\sup _{n} E_{\kappa_{n}}\left(w_{n}, B_{2}\right)<+\infty$. In view of Proposition 3.1, up to a further subsequence, $w_{n} \rightarrow \phi$ weakly in $H^{1}\left(B_{2} ; \mathbb{R}^{3}\right)$ where $\phi: B_{2} \rightarrow \mathbb{S}^{2}$ is a weakly harmonic map, and

$$
e_{\kappa_{n}}\left(w_{n}\right) d x \stackrel{*}{\rightarrow} \mu:=\frac{1}{2}|\nabla \phi|^{2} d x+v \quad \text { weakly* as measures on } B_{2},
$$

for some Radon measure $v=4 \pi \theta \mathcal{H}^{1}\left\llcorner\Sigma\right.$ where $\Sigma$ is a $\mathcal{H}^{1}$-rectifiable set with locally finite $\mathcal{H}^{1}$-measure and $\theta$ is an integer valued function. As a direct consequence of the Monotonicity Formula (1.7) and 3.7), we have

$$
\frac{1}{R} v\left(B_{R}\left(x_{0}\right)\right) \leq \frac{1}{R} \mu\left(B_{R}\left(x_{0}\right)\right) \leq 4 \pi
$$

for every $x_{0} \in B_{2}$ and $0<R<\operatorname{dist}\left(x_{0}, \partial B_{2}\right)$. By Theorem 2.83 in [4], the 1-dimensional density of $v$ at $x_{0}$, i.e., $\Theta_{1}\left(v, x_{0}\right)=\lim _{R \rightarrow 0}(2 R)^{-1} v\left(B_{R}\left(x_{0}\right)\right)$, exists and coincides with $4 \pi \theta\left(x_{0}\right)$ for $\mathcal{H}^{1}$-a.e. $x_{0} \in \Sigma$. In view of 3.9) we deduce that $\theta \leq 1 / 2 \mathcal{H}^{1}$-a.e. on $\Sigma$. Since $\theta$ is integer valued, we have $\theta=0 \mathcal{H}^{1}$-a.e. on $\Sigma$, i.e., $v \equiv 0$. Going back to (3.8), we conclude that $w_{n} \rightarrow \phi$ strongly in $H_{\text {loc }}^{1}\left(B_{2} ; \mathbb{R}^{3}\right)$ and

$$
\kappa_{n}^{2}\left(1-\left|w_{n}\right|^{2}\right)^{2} \stackrel{n \rightarrow+\infty}{\longrightarrow} 0 \quad \text { in } L_{\mathrm{loc}}^{1}\left(B_{2}\right) .
$$

It now remains to prove the stationarity of $\phi$. Since $w_{n}$ is smooth and satisfies 3.5, we have

$$
\int_{B_{2}} e_{\kappa_{n}}\left(w_{n}\right) \operatorname{div} \zeta-\sum_{i, j=1}^{3} \frac{\partial \zeta_{i}}{\partial x_{j}} \frac{\partial w_{n}}{\partial x_{i}} \cdot \frac{\partial w_{n}}{\partial x_{j}}=0
$$

for every $\zeta \in C_{c}^{1}\left(B_{2} ; \mathbb{R}^{3}\right)$. Using the local strong convergence of $w_{n}$ and 3.10 , we can let $n \rightarrow+\infty$ in the above equation to derive that

$$
\int_{B_{2}}|\nabla \phi|^{2} \operatorname{div} \zeta-2 \sum_{i, j=1}^{3} \frac{\partial \zeta_{i}}{\partial x_{j}} \frac{\partial \phi}{\partial x_{i}} \cdot \frac{\partial \phi}{\partial x_{j}}=0 \quad \forall \zeta \in C_{c}^{1}\left(B_{2} ; \mathbb{R}^{3}\right)
$$

i.e., $\phi$ is stationary in $B_{2}$.

Step 4. By the energy monotonicity formula for stationary harmonic maps (see [27]) and 3.7), we have

$$
\frac{1}{R_{1}} \int_{B_{R_{1}}\left(x_{0}\right)}|\nabla \phi|^{2} \leq \frac{1}{R_{2}} \int_{B_{R_{2}}\left(x_{0}\right)}|\nabla \phi|^{2} \leq 8 \pi
$$


for every $x_{0} \in B_{2}$ and $0<R_{1} \leq R_{2} \leq \operatorname{dist}\left(x_{0}, \partial B_{2}\right)$. We claim that

$$
\lim _{R \rightarrow 0} \frac{1}{R} \int_{B_{R}\left(P_{i}\right)}|\nabla \phi|^{2}=\inf _{0<R<1} \frac{1}{R} \int_{B_{R}\left(P_{i}\right)}|\nabla \phi|^{2}>0 \quad \text { for } i=1,2 .
$$

Indeed, if the limit above vanishes, we could argue as in Step 2 using Lemma 3.1 to deduce that $\left|w_{n}\left(P_{i}\right)\right|>\delta$ for $n$ large, which contradicts 3.6. By the quantization results in [20], for $i=1,2$,

$$
\lim _{R \rightarrow 0} \frac{1}{R} \int_{B_{R}\left(P_{i}\right)}|\nabla \phi|^{2}=8 \pi k_{i} \quad \text { for some } k_{i} \in \mathbb{N} .
$$

Combining 3.11 with 3.12, we deduce that $k_{1}=k_{2}=1$ and thus

$$
\inf _{0<R<1} \frac{1}{R} \int_{B_{R}\left(P_{i}\right)}|\nabla \phi|^{2}=8 \pi \quad \text { for } i=1,2 .
$$

Setting $Q_{R}=(R-1 / 2,0,0)$ for $0<R<1$, we then have

$$
8 \pi \geq \int_{B_{1}\left(Q_{R}\right)}|\nabla \phi|^{2} \geq \int_{B_{R}\left(P_{1}\right)}|\nabla \phi|^{2}+\int_{B_{1-R}\left(P_{2}\right)}|\nabla \phi|^{2} \geq 8 \pi R+8 \pi(1-R)=8 \pi .
$$

Hence $|\nabla \phi|^{2} \equiv 0$ a.e. in $B_{1}\left(Q_{R}\right) \backslash\left(B_{R}\left(P_{1}\right) \cup B_{1-R}\left(P_{2}\right)\right)$ for every $0<R<1$. Since

$$
B_{1} \cap \bigcup_{0<R<1}\left(B_{1}\left(Q_{R}\right) \backslash\left(B_{R}\left(P_{1}\right) \cup B_{1-R}\left(P_{2}\right)\right)\right)=B_{1} \backslash[(-1,0,0),(1,0,0)],
$$

we derive that $\int_{B_{1}}|\nabla \phi|^{2}=0$, which obviously contradicts 3.13 . Therefore $r_{n} \lambda_{n}$ remains bounded and the proof is complete.

Proof of Theorem 1.1 Consider a sequence $\lambda_{n} \rightarrow+\infty$ and let $u_{n}$ be a minimizer of $E_{\lambda_{n}}\left(\cdot, B_{1}\right)$ on $H_{\mathrm{Id}}^{1}\left(B_{1} ; \mathbb{R}^{3}\right)$. By Proposition $3.2,\left|u_{n}\right| \geq 1 / 2$ in $B_{1} \backslash B_{1 / 2}$ for $n$ large enough. In particular, $d_{r}:=\operatorname{deg}\left(u_{n}, \partial B_{r}\right)$ is well defined for $1 / 2 \leq r \leq 1$ and $d_{r}=d_{1}=1$ thanks to the boundary condition. Hence we may find $a_{n} \in B_{1 / 2}$ such that $u_{n}\left(a_{n}\right)=0$ for every $n$ sufficiently large. Again by Proposition 3.2, $a_{n} \rightarrow 0$ and $\left\{\left|u_{n}\right| \leq 1 / 2\right\} \subset B_{r_{n}}\left(a_{n}\right)$ with $r_{n}:=\operatorname{diam}\left(\left\{\left|u_{n}\right| \leq 1 / 2\right\}\right)=\mathcal{O}\left(\lambda_{n}^{-1}\right)$. Therefore $\operatorname{deg}\left(u_{n}, \partial B_{r}\left(a_{n}\right)\right)=1$ for any $r \in\left[r_{n}, 1 / 2\right]$.

Setting $R_{n}:=\lambda_{n}\left(1-\left|a_{n}\right|\right)$, we have $R_{n} \rightarrow+\infty$ as $n \rightarrow+\infty$, and we define $\bar{u}_{n}(x):=u_{n}\left(\lambda_{n}^{-1} x+a_{n}\right)$ for $x \in B_{R_{n}}$, so that $\bar{u}_{n}$ satisfies

$$
\Delta \bar{u}_{n}+\bar{u}_{n}\left(1-\left|\bar{u}_{n}\right|^{2}\right) \quad \text { in } B_{R_{n}},
$$

$\bar{u}_{n}(0)=0$ and $\left|\bar{u}_{n}\right| \leq 1$ for every $n$. Moreover, arguing as in the previous proof, we obtain

$$
\limsup _{n \rightarrow+\infty} R_{n}^{-1} E_{1}\left(\bar{u}_{n}, B_{R_{n}}\right) \leq 4 \pi \text {. }
$$

Then we infer from standard elliptic theory that, up to a subsequence, $\bar{u}_{n} \rightarrow u$ in $C_{\mathrm{loc}}^{2}\left(\mathbb{R}^{3}\right)$ for some map $u: \mathbb{R}^{3} \rightarrow \mathbb{R}^{3}$ solving $\Delta u+u\left(1-|u|^{2}\right)=0$ in $\mathbb{R}^{3}$ and $u(0)=0$. 
By Proposition 3.2 and the choice of $a_{n}$, we have $\left\{\left|\bar{u}_{n}\right| \leq 1 / 2\right\} \subset \bar{B}_{R_{0}}$ with $R_{0}:=$ $\sup _{n} \lambda_{n} r_{n}<+\infty$. Hence $|u| \geq 1 / 2$ in $\mathbb{R}^{3} \backslash B_{R_{0}}$ by continuity and locally uniform convergence. As a consequence, $u$ is nonconstant, $\operatorname{deg}_{\infty} u$ is well defined and

$$
\operatorname{deg}_{\infty} u=\operatorname{deg}\left(u, \partial B_{R}\right)=\lim _{n \rightarrow+\infty} \operatorname{deg}\left(\bar{u}_{n}, \partial B_{R}\right)=\lim _{n \rightarrow+\infty} \operatorname{deg}\left(u_{n}, \partial B_{r_{n}}\left(a_{n}\right)\right)=1
$$

for any $R \geq R_{0}$. Arguing in the same way, we infer from Proposition 3.2 that $|u(x)| \rightarrow 1$ as $|x| \rightarrow+\infty$. Next we deduce from 3.14, the Monotonicity Formula 1.7 and the smooth convergence of $\bar{u}_{n}$ to $u$ that $\sup _{R>0} R^{-1} E_{1}\left(u, B_{R}\right) \leq 4 \pi$. By the quantization result [22, Corollary D], we have $R^{-1} E_{1}\left(u, B_{R}\right) \rightarrow 4 \pi k$ as $R \rightarrow+\infty$ with $k \in\{0,1\}$. Since $u$ is nonconstant, we see that $k=1$. Finally, the local minimality of $u$ easily follows from the minimality of $u_{n}$ and the strong convergence in $H_{\text {loc }}^{1}\left(\mathbb{R}^{3} ; \mathbb{R}^{3}\right)$ of $\bar{u}_{n}$ to $u$.

\section{Energy quantization for local minimizers}

This section is devoted to the proof of Theorem 1.2. For any solution $u$ of (1.1) satisfying (1.8), the scaled maps $u_{R}(x):=u(R x)$ are relatively weakly compact in $H_{\text {loc }}^{1}\left(\mathbb{R}^{3} ; \mathbb{R}^{3}\right)$. This fact will allow us to study such a map $u$ near infinity. First we recall that a tangent map to $u$ at infinity is a map $\phi: \mathbb{R}^{3} \rightarrow \mathbb{R}^{3}$ obtained as a weak limit of $u_{n}(x):=u\left(x / R_{n}\right)$ in $H_{\text {loc }}^{1}\left(\mathbb{R}^{3} ; \mathbb{R}^{3}\right)$ for some sequence of radii $R_{n} \rightarrow+\infty$. We denote by $\mathcal{T}_{\infty}(u)$ the set of all possible tangent maps to $u$ at infinity. The only information given by the potential at infinity is that any $\phi \in \mathcal{T}_{\infty}(u)$ takes values in $\mathbb{S}^{2}$. This is any easy consequence of the following elementary lemma.

Lemma 4.1. Let $u \in H_{\mathrm{loc}}^{1}\left(\mathbb{R}^{3} ; \mathbb{R}^{3}\right)$ be a solution of 1.1 satisfying 1.8 . Then

$$
\lim _{R \rightarrow+\infty} \frac{1}{R} \int_{B_{R}} \frac{\left(1-|u|^{2}\right)^{2}}{4} d x=0 .
$$

Proof. We apply (1.7) with $\lambda=1, r>0$ and $R=2 r$ to obtain

$\frac{1}{r} \int_{B_{r}} \frac{\left(1-|u|^{2}\right)^{2}}{4} d x \leq 4 \int_{r}^{2 r} \frac{1}{t^{2}}\left(\int_{B_{t}} \frac{\left(1-|u|^{2}\right)^{2}}{4} d x\right) d t \leq \frac{1}{2 r} E\left(u, B_{2 r}\right)-\frac{1}{r} E\left(u, B_{r}\right)$.

Since the left hand side of $(1.7)$ is bounded and increasing, the right hand side above tends to zero as $r$ tends to infinity and the conclusion follows.

The following description of any tangent map has been obtained in [22, Theorem C].

Proposition 4.1. Let $u$ be a solution of (1.1) satisfying (1.8). Let $\phi \in \mathcal{T}_{\infty}(u)$ and let $R_{n} \rightarrow+\infty$ be an associated sequence of radii. Then $\phi(x)=\phi(x /|x|)$ for $x \neq 0$ and $\phi_{\mid \mathbb{S}^{2}}$ is a smooth harmonic map with values in $\mathbb{S}^{2}$. Moreover there exists a subsequence (not relabelled) such that

$$
e_{R_{n}}\left(u_{n}\right) d x \stackrel{*}{\rightarrow} \frac{1}{2}|\nabla \phi|^{2} d x+v \quad \text { as } n \rightarrow+\infty,
$$

weakly* as measures for some nonnegative Radon measure $v$. In addition, if $v \not \equiv 0$ there exists an integer $1 \leq l<\infty,\left\{P_{j}\right\}_{j=1}^{l} \subset \mathbb{S}^{2}$ and $\left\{k_{j}\right\}_{j=1}^{l} \subset \mathbb{N}^{*}$ such that 
(i) $\operatorname{spt}(v)=\bigcup_{j=1}^{l} \overline{O P_{j}}$ where $\overline{O P_{j}}$ denotes the ray emitting from the origin to $P_{j}$, and for $1 \leq j \leq l$,

$$
v\left\llcorner\overline{O P_{j}}=4 \pi k_{j} \mathcal{H}^{1}\left\llcorner\overline{O P_{j}} ;\right.\right.
$$

(ii) the following balancing condition holds:

$$
\frac{1}{2} \int_{\mathbb{S}^{2}} x|\nabla \phi|^{2} d \mathcal{H}^{2}+4 \pi \sum_{j=1}^{l} k_{j} P_{j}=0 .
$$

Under the assumption (1.8) we can apply Proposition 4.1 to any local minimizer of $E(\cdot)$. Now we claim that the local minimality of $u$ implies the strong convergence of the scaled maps $\left\{u_{n}\right\}$ to the associated tangent map.

Proposition 4.2. Let $u \in H_{\mathrm{loc}}^{1}\left(\mathbb{R}^{3} ; \mathbb{R}^{3}\right)$ be a local minimizer of $E(\cdot)$ satisfying 1.8 . Let $\phi \in \mathcal{T}_{\infty}(u)$ and let $R_{n} \rightarrow+\infty$ be the associated sequence of radii given by Proposition 4.1 Then $u_{n} \rightarrow \phi$ strongly in $H_{\mathrm{loc}}^{1}\left(\mathbb{R}^{3}\right)$ as $n \rightarrow+\infty$ and

$$
e_{R_{n}}\left(u_{n}\right) d x \stackrel{*}{\rightarrow} \frac{1}{2}|\nabla \phi|^{2} d x
$$

weakly* as measures.

Proof. In view of Proposition 4.1, it suffices to prove that the defect measure $v$ in (4.2) actually vanishes. We shall achieve this using a comparison argument. First we improve the convergence of $u_{n}$ away from $\operatorname{spt}(v)$.

Step 1. First observe that $R_{n}^{2}\left(1-\left|u_{n}\right|^{2}\right)^{2} \rightarrow 0$ in $L_{\text {loc }}^{1}\left(\mathbb{R}^{3}\right)$ by scaling and Lemma 4.1 Next we claim that $u_{n} \rightarrow \phi$ in $C_{\text {loc }}^{1}\left(\mathbb{R}^{3} \backslash(\operatorname{spt}(v) \cup\{0\})\right)$. Fix a ball $B_{4 \delta}\left(x_{0}\right) \subset \subset \mathbb{R}^{3} \backslash$ $(\operatorname{spt}(\nu) \cup\{0\})$ with arbitrary center and $\delta$ to be chosen. Since $\phi$ is smooth away from the origin, we can choose $\delta$ small such that $\int_{B_{4 \delta}\left(x_{0}\right)}|\nabla \phi|^{2}<4 \delta \eta_{0}$ where the constant $\eta_{0}$ is given by Lemma 3.1. In view of (4.2), we have $\int_{B_{4 \delta}\left(x_{0}\right)} e_{R_{n}}\left(u_{n}\right) \rightarrow \frac{1}{2} \int_{B_{4 \delta}\left(x_{0}\right)}|\nabla \phi|^{2}$. In particular $\int_{B_{4 \delta}\left(x_{0}\right)} e_{R_{n}}\left(u_{n}\right) \leq 4 \delta \eta_{0}$ for $n$ large enough. By Lemma 3.1, we infer that $\left|\nabla u_{n}\right| \leq C_{\delta, x_{0}}$ and $\left|u_{n}\right| \geq 1 / 2$ in $B_{2 \delta}\left(x_{0}\right)$ for $n$ large and a constant $C_{\delta, x_{0}}$ independent of $n$. Since $u_{n}$ satisfies (1.10) (with $R=R_{n}$ ), setting $\rho_{n}:=1-\left|u_{n}\right|^{2}$, we have $0 \leq \rho_{n} \leq 1$ and $-\Delta \rho_{n}+R_{n}^{2} \rho_{n} \leq 2 C_{\delta, x_{0}}^{2}$ in $B_{2 \delta}\left(x_{0}\right)$. By a slight modification of Lemma 2 in [6], we infer that $\rho_{n} \leq C_{\delta, x_{0}}^{\prime} R_{n}^{-2}$ in $B_{\delta}\left(x_{0}\right)$ for some constant $C_{\delta, x_{0}}^{\prime}$ independent of $n$. Going back to 1.10 we deduce that $\left|\Delta u_{n}\right| \leq C_{\delta, x_{0}}^{\prime}$ in $B_{\delta}\left(x_{0}\right)$. Using standard $W_{\text {loc }}^{2, p}$-regularity and the Sobolev embedding in $C^{1, \alpha}$-spaces, we finally conclude that $u_{n} \rightarrow \phi$ in $C^{1}\left(B_{\delta / 2}\left(x_{0}\right)\right)$.

Step 2. We argue by contradiction and assume that $v \not \equiv 0$ so that $k_{1} \geq 1$. Without loss of generality we may also assume that $P_{1}=(1,0,0)$ and $\phi\left(P_{1}\right)=(0,0,1)=: N$. We will construct for $n$ sufficiently large comparison maps $w_{n}$ which, roughly speaking, agree with $u_{n}$ except in a small cylinder around the $x_{1}$ axis, where they are constantly equal to $N$ and with smaller energy. We consider two small parameters $0<\delta \ll 1$ and 
$0<\sigma \ll 1$. In view of the explicit form of $\phi$ and $v$, we can find $x_{\sigma} \in \overline{O P_{1}}$ with $\left|x_{\sigma}\right|$ as large as needed such that $\bar{Q}_{4}\left(x_{\sigma}\right) \cap \overline{O P_{j}}=\emptyset$ for each $2 \leq j \leq l$,

$$
\phi\left(Q_{4}\left(x_{\sigma}\right)\right) \subset B_{\sigma}(N) \quad \text { and } \quad \int_{Q_{4}\left(x_{\sigma}\right)}|\nabla \phi|^{2}<\sigma .
$$

Here we use the notation $Q_{\rho}\left(x_{\sigma}\right)=x_{\sigma}+\rho(-1 / 2,1 / 2)^{3}$ for $\rho>0$. Throughout the proof $T_{\delta}:=\mathbb{R} \times B_{\delta}^{(2)}(0) \subset \mathbb{R}^{3}$ will denote the infinite cylinder of size $\delta$ around the $x_{1}$ axis. In view of Step 1, for $n$ large enough,

$$
\left|u_{n}-\phi\right|<\sigma \quad \text { in } Q_{4}\left(x_{\sigma}\right) \backslash T_{\delta / 2},
$$

and in particular $\left|u_{n}\right|$ does not vanish in $Q_{4}\left(x_{\sigma}\right) \backslash T_{\delta / 2}$ and it is actually as close to one as we want.

Consider a cut-off function $\chi_{1} \in C_{c}^{\infty}\left(Q_{4}\left(x_{\sigma}\right) ;[0,1]\right)$ satisfying $\chi_{1} \equiv 1$ in $Q_{3}\left(x_{\sigma}\right)$ and set $\psi_{\delta}(x):=\min \left\{\delta^{-1} \chi_{1}(x)\left(2\left|x^{\prime}\right|-\delta\right)^{+}, 1\right\}$ using the notation $x=\left(x_{1}, x^{\prime}\right)$. Then we define, for $x \in Q_{4}\left(x_{\sigma}\right)$,

$$
\bar{u}_{n}(x):=\psi_{\delta}(x) \frac{u_{n}(x)}{\left|u_{n}(x)\right|}+\left(1-\psi_{\delta}(x)\right) u_{n}(x) .
$$

Note that $\bar{u}_{n}=u_{n}$ in a neighborhood of $\partial Q_{4}\left(x_{\sigma}\right), \bar{u}_{n}=u_{n}$ in $Q_{4}\left(x_{\sigma}\right) \cap T_{\delta / 2}$, and $\left(1-\left|\bar{u}_{n}\right|^{2}\right)^{2} \leq\left(1-\left|u_{n}\right|^{2}\right)^{2}$, because the double well potential is locally convex near its minima. Then we easily infer from Step 1 that $\bar{u}_{n} \rightarrow \phi$ in $W^{1, \infty}\left(Q_{4}\left(x_{\sigma}\right) \backslash T_{\delta / 2}\right)$ and

$$
e_{R_{n}}\left(\bar{u}_{n}\right) d x\left\llcornerQ _ { 4 } ( x _ { \sigma } ) \stackrel { * } { \rightarrow } \frac { 1 } { 2 } | \nabla \phi | ^ { 2 } d x \left\llcorner Q_{4}\left(x_{\sigma}\right)+v\left\llcorner Q_{4}\left(x_{\sigma}\right)\right.\right.\right.
$$

weakly* as measures. Now consider a second cut-off function $\chi_{2} \in C_{c}^{\infty}\left(Q_{3}\left(x_{\sigma}\right) ;[0,1]\right)$ satisfying $\chi_{2} \equiv 1$ in $Q_{2}\left(x_{\sigma}\right)$ and set $\tilde{\psi}_{\delta}(x)=\min \left\{\delta^{-1} \chi_{2}(x)\left(\left|x^{\prime}\right|-\delta\right)^{+}, 1\right\}$. Define, for $x \in Q_{4}\left(x_{\sigma}\right)$,

$v_{n}(x):= \begin{cases}\frac{\tilde{\psi}_{\delta}(x) N+\left(1-\tilde{\psi}_{\delta}(x)\right) \bar{u}_{n}(x)}{\left|\tilde{\psi}_{\delta}(x) N+\left(1-\tilde{\psi}_{\delta}(x)\right) \bar{u}_{n}(x)\right|} & \text { if } x \in Q_{3}\left(x_{\sigma}\right) \backslash T_{\delta}, \\ \bar{u}_{n}(x) & \text { if } x \in\left(Q_{4}\left(x_{\sigma}\right) \backslash Q_{3}\left(x_{\sigma}\right)\right) \cup\left(Q_{4}\left(x_{\sigma}\right) \cap T_{\delta}\right),\end{cases}$

and

$$
\phi_{\delta}(x):=\frac{\tilde{\psi}_{\delta}(x) N+\left(1-\tilde{\psi}_{\delta}(x)\right) \phi(x)}{\left|\tilde{\psi}_{\delta}(x) N+\left(1-\tilde{\psi}_{\delta}(x)\right) \phi(x)\right|} .
$$

Note that $\phi_{\delta}$ and $v_{n}$ are well defined and smooth (Lipschitz) thanks to (4.4) and (4.5). Moreover $v_{n}=u_{n}$ both in a neighborhood of $\partial Q_{4}\left(x_{\sigma}\right)$ and in $Q_{4}\left(x_{\sigma}\right) \cap T_{\delta / 2}$, and $v_{n} \equiv N$ in $Q_{2}\left(x_{\sigma}\right) \backslash T_{2 \delta}$. From the construction of $\bar{u}_{n}$, we derive that $v_{n} \rightarrow \phi_{\delta}$ in $W^{1, \infty}\left(Q_{4}\left(x_{\sigma}\right) \backslash T_{\delta / 2}\right)$ and

$$
e_{R_{n}}\left(v_{n}\right) d x\left\llcornerQ _ { 4 } ( x _ { \sigma } ) \stackrel { * } { \rightarrow } \frac { 1 } { 2 } | \nabla \phi _ { \delta } | ^ { 2 } d x \left\llcorner Q_{4}\left(x_{\sigma}\right)+v\left\llcorner Q_{4}\left(x_{\sigma}\right)\right.\right.\right.
$$


weakly* as measures. Since $\nu$ does not charge the boundary of $Q_{\rho}\left(x_{\sigma}\right)$ for every $\rho>0$,

$$
\int_{\left|x_{\sigma}\right|+1 / 2}^{\left|x_{\sigma}\right|+1}\left(\int_{\left\{x_{1}=r\right\} \cap T_{2 \delta}} e_{R_{n}}\left(v_{n}\right)\right) d r \stackrel{n \rightarrow+\infty}{\longrightarrow} \frac{1}{2} \int_{\left\{\left|x_{\sigma}\right|+1 / 2<x_{1}<\left|x_{\sigma}\right|+1\right\} \cap T_{2 \delta}}\left|\nabla \phi_{\delta}\right|^{2}+2 \pi k_{1} .
$$

On the other hand, one may derive from the explicit form of $\phi_{\delta}$ and (4.4) that

$$
\int_{Q_{4}\left(x_{\sigma}\right)}\left|\nabla \phi_{\delta}\right|^{2} \leq C_{\delta} \sigma
$$

where $C_{\delta}$ denotes a constant independent of $\sigma$. Hence we can find $r_{n}^{+} \in\left[\left|x_{\sigma}\right|+1 / 2\right.$, $\left.\left|x_{\sigma}\right|+1\right]$ such that

$$
\limsup _{n \rightarrow+\infty} \int_{\left\{x_{1}=r_{n}^{+}\right\} \cap T_{2 \delta}} e_{R_{n}}\left(v_{n}\right) \leq 4 \pi k_{1}+C_{\delta} \sigma .
$$

Arguing in the same way, we find $r_{n}^{-} \in\left[\left|x_{\sigma}\right|-1,\left|x_{\sigma}\right|-1 / 2\right]$ such that

$$
\limsup _{n \rightarrow+\infty} \int_{\left\{x_{1}=r_{n}^{-}\right\} \cap T_{2 \delta}} e_{R_{n}}\left(v_{n}\right) \leq 4 \pi k_{1}+C_{\delta} \sigma .
$$

Next we introduce the sets

$$
\begin{aligned}
& C_{n}^{+}:=T_{2 \delta} \cap\left\{r_{n}^{+}-2 \delta \leq x_{1} \leq r_{n}^{+},\left|x^{\prime}\right| \leq x_{1}-\left(r_{n}^{+}-2 \delta\right)\right\}, \\
& C_{n}^{-}:=T_{2 \delta} \cap\left\{r_{n}^{-} \leq x_{1} \leq r_{n}^{-}+2 \delta,\left|x^{\prime}\right| \leq\left(r_{n}^{-}+2 \delta\right)-x_{1}\right\}, \\
& D_{n}:=T_{2 \delta} \cap\left\{x \in T_{2 \delta}, x_{1} \in\left(r_{n}^{-}, r_{n}^{+}\right)\right\} .
\end{aligned}
$$

Define, for $x \in Q_{4}\left(x_{\sigma}\right)$ and $n$ large enough,

$$
w_{n}(x)= \begin{cases}v_{n}(x) & \text { if } x \in Q_{4}\left(x_{\sigma}\right) \backslash D_{n}, \\ v_{n}\left(r_{n}^{+}, \frac{2 \delta x^{\prime}}{x_{1}-\left(r_{n}^{+}-2 \delta\right)}\right) & \text { if } x \in C_{n}^{+}, \\ v_{n}\left(r_{n}^{-}, \frac{2 \delta x^{\prime}}{\left(r_{n}^{-}+2 \delta\right)-x_{1}}\right) & \text { if } x \in C_{n}^{-}, \\ N & \text { if } x \in D_{n} \backslash\left(C_{n}^{+} \cup C_{n}^{-}\right) .\end{cases}
$$

One can check that $w_{n} \in H^{1}\left(Q_{4}\left(x_{\sigma}\right) ; \mathbb{R}^{3}\right)$ and $w_{n}=u_{n}$ in a neighborhood of $\partial Q_{4}\left(x_{\sigma}\right)$. Moreover, straightforward computations yield

$$
\int_{C_{n}^{+}} e_{R_{n}}\left(w_{n}\right) \leq C \delta \int_{\left\{x_{1}=r_{n}^{+}\right\} \cap T_{2 \delta}} e_{R_{n}}\left(v_{n}\right) \text { and } \int_{C_{n}^{-}} e_{R_{n}}\left(w_{n}\right) \leq C \delta \int_{\left\{x_{1}=r_{n}^{-}\right\} \cap T_{2 \delta}} e_{R_{n}}\left(v_{n}\right)
$$

for some absolute constant $C$. Recalling (4.6), (4.7), the fact that $v$ does not charge the boundary of $Q_{\rho}\left(x_{\sigma}\right)$ for every $\rho>0$ and $Q_{4}\left(x_{\sigma}\right)=\left(Q_{4}\left(x_{\sigma}\right) \backslash D_{n}\right) \cup\left(C_{n}^{+} \cup C_{n}^{-}\right) \cup$ $\left(D_{n} \backslash\left(C_{n}^{+} \cup C_{n}^{-}\right)\right)$, we finally obtain

$$
\limsup _{n \rightarrow+\infty} \int_{Q_{4}\left(x_{\sigma}\right)} e_{R_{n}}\left(w_{n}\right) \leq 12 \pi k_{1}+C \delta+C_{\delta} \sigma,
$$

for some constant $C$ independent $\sigma$ and $\delta$, and some constant $C_{\delta}$ independent of $\sigma$. 
Step 3. From the local minimality of $u$, we infer that

$$
\int_{Q_{4}\left(x_{\sigma}\right)} e_{R_{n}}\left(u_{n}\right) \leq \int_{Q_{4}\left(x_{\sigma}\right)} e_{R_{n}}\left(w_{n}\right) .
$$

Using (4.2) and 4.8 we let $n \rightarrow+\infty$ in the above inequality to derive

$$
\begin{aligned}
16 \pi k_{1} & \leq v\left(Q_{4}\left(x_{\sigma}\right)\right)+\int_{Q_{4}\left(x_{\sigma}\right)} \frac{1}{2}|\nabla \phi|^{2} d x=\lim _{n \rightarrow \infty} \int_{Q_{4}\left(x_{\sigma}\right)} e_{R_{n}}\left(u_{n}\right) \\
& \leq 12 \pi k_{1}+C \delta+C_{\delta} \sigma .
\end{aligned}
$$

Passing successively to the limits $\sigma \rightarrow 0$ and $\delta \rightarrow 0$, we conclude that $k_{1}=0$. This contradicts our assumption $k_{1} \geq 1$ and the proof is complete.

Corollary 4.1. Let $u \in H_{\mathrm{loc}}^{1}\left(\mathbb{R}^{3} ; \mathbb{R}^{3}\right)$ be a nonconstant local minimizer of $E(\cdot)$ satisfying 1.8. Then any $\phi \in \mathcal{T}_{\infty}(u)$ is of the form $\phi(x)=T x /|x|$ for some $T \in O(3)$.

Proof. Step 1. First we claim that any $\phi \in \mathcal{T}_{\infty}(u)$ is energy minimizing in $B_{1}$, i.e.,

$$
\int_{B_{1}}|\nabla \phi|^{2} d x \leq \int_{B_{1}}|\nabla \varphi|^{2} d x \quad \text { for all } \varphi \in H^{1}\left(B_{1} ; S^{2}\right) \text { such that } \varphi_{\mid \partial B_{1}}=\phi .
$$

Let $R_{n} \rightarrow+\infty$ be the sequence of radii given by Proposition 4.1 , and let $\left\{u_{n}\right\}$ be the associated sequence of scaled maps. It follows from Step 2 in the previous proof that

$$
\int_{B_{1}} e_{R_{n}}\left(u_{n}\right) d x \rightarrow \frac{1}{2} \int_{B_{1}}|\nabla \phi|^{2} d x
$$

as $n \rightarrow+\infty$. In particular,

$$
R_{n}^{2} \int_{B_{1}}\left(1-\left|u_{n}\right|^{2}\right)^{2} d x \rightarrow 0
$$

In view of the local minimality of $u$, it suffices to prove that for any $\varphi \in H_{\phi}^{1}\left(B_{1} ; S^{2}\right)$, there exists a sequence $\varphi_{n} \in H_{u_{n}}^{1}\left(B_{1} ; \mathbb{R}^{3}\right)$ such that

$$
\int_{B_{1}} e_{R_{n}}\left(\varphi_{n}\right) d x \rightarrow \frac{1}{2} \int_{B_{1}}|\nabla \varphi|^{2} d x .
$$

We proceed as follows. From the previous proof we know that $u_{n} \rightarrow \phi$ uniformly in the annulus $K:=\bar{B}_{1} \backslash B_{1 / 2}$. In particular, $\left|u_{n}\right| \geq 1 / 2$ in $K$ for $n$ large, and setting $v_{n}:=u_{n} /\left|u_{n}\right|$ we obtain

$$
\delta_{n}:=\left\|v_{n}-\phi\right\|_{L^{\infty}(K)}+\left\|1-\left|u_{n}\right|^{2}\right\|_{L^{\infty}(K)} \stackrel{n \rightarrow+\infty}{\longrightarrow} 0 .
$$

Denote $\mathcal{D}:=\left\{\left(s_{0}, s_{1}\right) \in \mathbb{S}^{2} \times \mathbb{S}^{2}:\left|s_{0}-s_{1}\right|<1 / 4\right\}$ and consider a continuously differentiable mapping $\Pi: \mathcal{D} \times[0,1] \rightarrow \mathbb{S}^{2}$ satisfying

$$
\Pi\left(s_{0}, s_{1}, 0\right)=s_{0}, \quad \Pi\left(s_{0}, s_{1}, 1\right)=s_{1}, \quad\left|\frac{\partial \Pi}{\partial t}\left(s_{0}, s_{1}, t\right)\right| \leq C\left|s_{0}-s_{1}\right|,
$$

e.g., the map giving geodesic convex combinations between points $s_{0}$ and $s_{1}$ on $\mathbb{S}^{2}$. 
Given $\varphi \in H_{\phi}^{1}\left(B_{1} ; S^{2}\right)$, we define, for $n$ large enough,

$$
\varphi_{n}(x)= \begin{cases}\varphi\left(\frac{x}{1-2 \delta_{n}}\right) & \text { for } x \in B_{1-2 \delta_{n},} \\ \Pi\left(v_{n}(x), \phi(x), \frac{1-\delta_{n}-|x|}{\delta_{n}}\right) & \text { for } x \in B_{1-\delta_{n}} \backslash B_{1-2 \delta_{n}}, \\ \left(\frac{1-|x|}{\delta_{n}}+\left|u_{n}(x)\right| \frac{|x|-1+\delta_{n}}{\delta_{n}}\right) v_{n}(x) & \text { for } x \in B_{1} \backslash B_{1-\delta_{n}} .\end{cases}
$$

One can easily check that $\varphi_{n} \in H^{1}\left(B_{1} ; \mathbb{R}^{3}\right)$ and that

$$
\begin{aligned}
\int_{B_{1}} e_{R_{n}}\left(\varphi_{n}\right) d x= & \frac{1-2 \delta_{n}}{2} \int_{B_{1}}|\nabla \varphi|^{2} d x+\frac{1}{2} \int_{B_{1-\delta_{n}} \backslash B_{1-2 \delta_{n}}}\left|\nabla \varphi_{n}\right|^{2} d x \\
& +\int_{B_{1} \backslash B_{1-\delta_{n}}} e_{R_{n}}\left(\varphi_{n}\right) d x .
\end{aligned}
$$

Straightforward computations yield

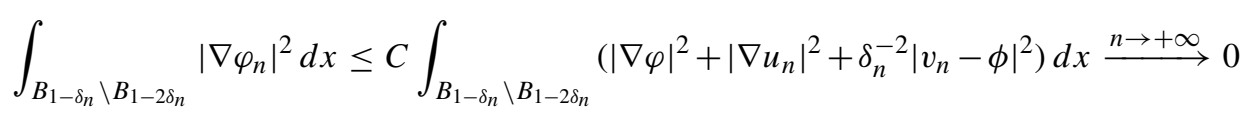

and

$$
\int_{B_{1} \backslash B_{1-\delta_{n}}} e_{R_{n}}\left(\varphi_{n}\right) d x \leq C \int_{B_{1} \backslash B_{1-\delta_{n}}}\left(\left|\nabla u_{n}\right|^{2}+\left(\delta_{n}^{-2}+R_{n}^{2}\right)\left(1-\left|u_{n}\right|^{2}\right)^{2}\right) d x \stackrel{n \rightarrow+\infty}{\longrightarrow} 0,
$$

where we used the fact that $\left(1-\left|\varphi_{n}\right|^{2}\right)^{2} \leq\left(1-\left|u_{n}\right|^{2}\right)^{2}$ for $n$ large enough, again by convexity of the double well potential near its minima, and 4.10) in the last estimate. In view of 4.12, this completes the proof of 4.11).

Step 2. In view of the monotonicity with respect to $R$ of $R^{-1} E\left(u, B_{R}\right)$, if $u$ is nonconstant then 4.3 yields

$$
0<\lim _{R \rightarrow+\infty} R^{-1} E\left(u, B_{R}\right)=\lim _{n \rightarrow+\infty} E_{R_{n}}\left(u_{n}, B_{1}\right)=\frac{1}{2} \int_{B_{1}}|\nabla \phi|^{2} d x
$$

and thus $\phi$ is nonconstant. Then the conclusion follows from Theorems 7.3 and 7.4 in [8] together with 4.9 .

Proof of Theorem 1.2. Let $R_{n} \rightarrow+\infty$ be an arbitrary sequence of radii. By (1.8), Propositions 4.1 and 4.2 and Corollary 4.1, we can find a subsequence (not relabelled) and $T \in O(3)$ such that the sequence of scaled maps $u_{n}(x)=u\left(R_{n} x\right)$ converges strongly in $H_{\text {loc }}^{1}\left(\mathbb{R}^{3} ; \mathbb{R}^{3}\right)$ to $\phi(x)=T x /|x|$. Therefore 4.13 gives $R^{-1} E\left(u, B_{R}\right) \rightarrow 4 \pi$ as $R \rightarrow+\infty$, and the proof is complete. 


\section{Asymptotic symmetry}

In order to study the asymptotic behavior of local minimizers we first derive some decay properties of solutions to (1.1) at infinity. It will be clear that the crucial ingredients are 1.8 , the $H_{\text {loc }}^{1}\left(\mathbb{R}^{3} ; \mathbb{R}^{3}\right)$ compactness of the scaled maps and the small energy regularity lemma recalled in Section 3. Then we bootstrap the first order estimates to get higher order estimates and compactness of the rescaled maps and their derivatives of all orders. Finally we prove a decay property of the radial derivative which will give uniqueness of the asymptotic limit at infinity in the $L^{2}$-topology, whence uniqueness of the limit in any topology follows.

We start with the following result.

Proposition 5.1. Let $u$ be a smooth solution to (1.1) satisfying (1.8) and such that the scaled maps $\left\{u_{R}\right\}_{R>0}$ are relatively compact in $H_{\text {loc }}^{1}\left(\mathbb{R}^{3} ; \mathbb{R}^{3}\right)$. Then there is a constant $C>0$ such that for all $x \in \mathbb{R}^{3}$,

$$
|x|^{2}\left(1-|u(x)|^{2}\right)+|x||\nabla u(x)| \leq C .
$$

Proof. We prove the statement by contradiction. Assume (5.1) were false; then there would be a sequence $\left\{x_{n}\right\} \subset \mathbb{R}^{3}$ such that $R_{n}=\left|x_{n}\right| \rightarrow+\infty$ as $n \rightarrow+\infty$ and

$$
\left|x_{n}\right|\left|\nabla u\left(x_{n}\right)\right|+\left|x_{n}\right|^{2}\left(1-\left|u\left(x_{n}\right)\right|^{2}\right) \stackrel{n \rightarrow+\infty}{\longrightarrow}+\infty .
$$

For each integer $n$, let us consider $u_{n}(x):=u_{R_{n}}(x)=u\left(R_{n} x\right)$ as an entire solution of (1.10). Taking a subsequence, we may assume that $x_{n} / R_{n} \rightarrow \bar{x} \in \partial B_{1}$ as $n \rightarrow+\infty$. By Proposition 4.1, up to a further subsequence the sequence $\left\{u_{n}\right\}$ of scaled maps converges to $u_{\infty}(x)=\omega(x /|x|)$ strongly in $H_{\text {loc }}^{1}\left(\mathbb{R}^{3} ; \mathbb{R}^{3}\right)$ as $n \rightarrow+\infty$, where $\omega: \mathbb{S}^{2} \rightarrow \mathbb{S}^{2}$ is a harmonic map. In addition $e_{R_{n}}\left(u_{n}\right)(x) d x \stackrel{*}{\rightarrow} \frac{1}{2}\left|\nabla u_{\infty}\right|^{2} d x+v$ where $v$ is a quantized cone-measure. Combining this property with the strong convergence in $H_{\text {loc }}^{1}\left(\mathbb{R}^{3} ; \mathbb{R}^{3}\right)$ and Lemma 4.1. we conclude that $v \equiv 0$. Since $\omega$ is a smooth map we have $u_{\infty} \in C^{\infty}\left(\mathbb{R}^{3} \backslash\right.$ $\left.\{0\} ; \mathbb{S}^{2}\right)$. In particular $u_{\infty}$ is smooth around $\bar{x} \in \partial B_{1}$. Now we can argue as in Step 1 in the proof of Proposition 4.2 to find $\delta>0$ such that $\left|\nabla u_{n}\right|+R_{n}^{2}\left(1-\left|u_{n}\right|^{2}\right) \leq C_{\delta}$ in $B_{\delta}(\bar{x})$ for some constant $C_{\delta}$ independent of $n$. Scaling back we obtain, for $n$ large enough,

$$
\left|x_{n}\right|\left|\nabla u\left(x_{n}\right)\right|+\left|x_{n}\right|^{2}\left(1-\left|u\left(x_{n}\right)\right|^{2}\right) \leq C_{\delta},
$$

which obviously contradicts 5.2 .

Remark 5.1. For an arbitrary entire solution $u$ to (1.1), the estimate 5.1) still holds under the assumption $|u(x)|=1+\mathcal{O}\left(|x|^{-2}\right)$ as $|x| \rightarrow+\infty$. Indeed, since the scaled map $u_{R}$ given by 1.9 satisfies 1.10, $\left\{\Delta u_{R}\right\}_{R>0}$ is equibounded in $L_{\text {loc }}^{\infty}\left(\mathbb{R}^{3} \backslash\{0\}\right)$. Therefore standard $W_{\text {loc }}^{2, p}$ estimates and the Sobolev embedding show that $\left\{\nabla u_{R}\right\}_{R>0}$ is equibounded in $L_{\text {loc }}^{\infty}\left(\mathbb{R}^{3} \backslash\{0\}\right)$, which proves 5.1 . Note also that 5.1 implies 1.8 . 
For a solution $u$ to (1.1) satisfying the assumptions of Proposition 5.1, we have $|u(x)|=1+\mathcal{O}\left(|x|^{-2}\right)$ and $|\nabla u(x)|=\mathcal{O}\left(|x|^{-1}\right)$ as $|x| \rightarrow+\infty$. In order to get bounds on the higher order derivatives of $u$ at infinity it is very convenient to use the polar decomposition for $u$, i.e., to write $u=\rho w$ for some nonnegative function $\rho$ and some $\mathbb{S}^{2}$-valued map $w$. The following result gives the 3D counterpart of the asymptotic estimates of [28] for the $2 \mathrm{D}$ case, and it is essentially based on the techniques introduced in the proof of [5, Theorem 1].

Proposition 5.2. Let $u$ be an entire solution of (1.1) satisfying (5.1). Let $R_{0} \geq 1$ be such that $|u(x)| \geq 1 / 2$ for $|x| \geq R_{0} / 4$. For $R \geq R_{0}$ and $|x| \geq 1 / 4$, let $u_{R}(x)=u(R x)=$ $\rho_{R}(x) w_{R}(x)$ be the polar decomposition of the scaled maps, i.e., $\rho_{R}(x):=\left|u_{R}(x)\right|$ and $w_{R}(x):=u_{R}(x) /\left|u_{R}(x)\right|$. Then for each $k \in \mathbb{N}$ and each $\sigma \in(1,2)$ there exist constants $C=C(k, \sigma)>0$ and $C^{\prime}=C^{\prime}(k, \sigma)>0$ independent of $R$ such that

$$
\begin{array}{ll}
\left(\mathcal{P}_{k}^{\prime}\right) & \left\|\nabla w_{R}\right\|_{C^{k}\left(\bar{B}_{2 \sigma} \backslash B_{1 / 2 \sigma}\right)} \leq C^{\prime}(k, \sigma), \\
\left(\mathcal{P}_{k}^{\prime \prime}\right) & \left\|R^{2}\left(1-\rho_{R}\right)\right\|_{C^{k}\left(\bar{B}_{2 \sigma} \backslash B_{1 / 2 \sigma}\right)} \leq C^{\prime \prime}(k, \sigma) .
\end{array}
$$

As a consequence, for each $k \in \mathbb{N}$ there is a constant $C(k)>0$ such that

$$
\sup _{x \in \mathbb{R}^{3}}\left(|x|^{k+1}\left|\nabla^{k+1} u(x)\right|+|x|^{k+2}\left|\nabla^{k}\left(1-|u(x)|^{2}\right)\right|\right) \leq C(k) .
$$

Proof. Observe that it suffices to prove (5.3) since (5.4) follows by scaling. For $|x| \geq$ $R_{0} / 4$ we have $|u(x)| \geq 1 / 2$ so we can write $u(x)=\rho(x) w(x)$ with $\rho(x):=|u(x)|$ and $w(x):=u(x) \rho(x)^{-1}$ and the system 1.12 is satisfied in $\mathbb{R}^{3} \backslash \bar{B}_{R_{0} / 4}$. Hence, for each $R \geq R_{0}$ the scaled maps $u_{R}, \rho_{R}$ and $w_{R}$ are well defined and smooth in $\mathbb{R}^{3} \backslash \bar{B}_{1 / 4}$. In addition, 1.12 yields by scaling the following Euler-Lagrange equations:

$$
\left\{\begin{array}{l}
\operatorname{div}\left(\rho_{R}^{2} \nabla w_{R}\right)+w_{R} \rho_{R}^{2}\left|\nabla w_{R}\right|^{2}=0 \\
\Delta \rho_{R}+\rho_{R} R^{2}\left(1-\rho_{R}^{2}\right)=\rho_{R}\left|\nabla w_{R}\right|^{2}
\end{array} \quad \text { in } \mathbb{R}^{3} \backslash \bar{B}_{1 / 4} .\right.
$$

We will prove 5.3 by induction on $k$, the case $k=0$ being easily true by assumption (5.1). We closely follow [5, pp. 136-137] with minor modifications.

First we prove that $\left(\mathcal{P}_{k}^{\prime}\right)-\left(\mathcal{P}_{k}^{\prime \prime}\right)$ implies $\left(\mathcal{P}_{k+1}^{\prime}\right)$. We set for simplicity

$$
X_{R}:=R^{2}\left(1-\rho_{R}\right),
$$

so that the second equation in 5.5 can be rewritten as

$$
-\Delta \rho_{R}=-\rho_{R}\left|\nabla w_{R}\right|^{2}+\rho_{R}\left(1+\rho_{R}\right) X_{R}
$$

By the inductive assumptions (5.3) the right hand side in 5.7) is bounded in $C_{\text {loc }}^{k}\left(B_{4} \backslash \bar{B}_{1 / 4}\right)$ uniformly with respect to $R \geq R_{0}$. Hence $\left\{\rho_{R}\right\}_{R \geq R_{0}}$ is bounded in $W_{\text {loc }}^{k+2, p}\left(B_{4} \backslash \bar{B}_{1 / 4}\right)$ for each $p<+\infty$ by standard elliptic regularity theory. Then the 
Sobolev embedding implies that $\left\{\nabla \rho_{R}\right\}_{R \geq R_{0}}$ is also bounded in $C_{\text {loc }}^{k}\left(B_{4} \backslash \bar{B}_{1 / 4}\right)$. Next rewrite the first equation in 5.5 as

$$
-\Delta w_{R}=w_{R}\left|\nabla w_{R}\right|^{2}+\frac{2 \nabla \rho_{R}}{\rho_{R}} \nabla w_{R}
$$

Since all the terms on the right hand side in $(5.8)$ are now bounded in $C_{\text {loc }}^{k}\left(B_{4} \backslash \bar{B}_{1 / 4}\right)$ uniformly with respect to $R \geq R_{0}$, standard linear theory (differentiating the equation $k$ times) also implies that $\left\{w_{R}\right\}_{R \geq R_{0}}$ is equibounded in $W_{\text {loc }}^{k+2, p}\left(B_{4} \backslash \bar{B}_{1 / 4}\right)$ for each $p<+\infty$. Therefore the right hand side in 5.8$)$ is in fact bounded in $W_{\text {loc }}^{k+1, p}\left(B_{4} \backslash \bar{B}_{1 / 4}\right)$ uniformly with respect to $R \geq R_{0}$. Hence the linear $L^{p}$-theory yields the boundedness of $\left\{w_{R}\right\}_{R \geq R_{0}}$ in $W_{\text {loc }}^{k+3, p}\left(B_{4} \backslash \bar{B}_{1 / 4}\right)$ for each $p<+\infty$. Then, by the Sobolev embedding, $\left\{\nabla w_{R}\right\}_{R \geq R_{0}}$ is bounded in $C_{\text {loc }}^{k+1}\left(B_{4} \backslash \bar{B}_{1 / 4}\right)$, i.e., $\left(\mathcal{P}_{k+1}^{\prime}\right)$ holds.

Now we prove that $\left(\mathcal{P}_{k}^{\prime}\right)-\left(\mathcal{P}_{k}^{\prime \prime}\right)$ implies $\left(\mathcal{P}_{k+1}^{\prime \prime}\right)$. We fix $\sigma \in(1,2)$ and we apply $\left(\mathcal{P}_{k}^{\prime}\right)$, $\left(\mathcal{P}_{k}^{\prime \prime}\right)$ and $\left(\mathcal{P}_{k+1}^{\prime}\right)$ in $\bar{B}_{2 \sigma^{\prime}} \backslash B_{1 / 2 \sigma^{\prime}}$ for a fixed $\sigma<\sigma^{\prime}<2$, e.g., $\sigma^{\prime}:=1+\sigma / 2$. Since $K:=\bar{B}_{2 \sigma} \backslash B_{1 / 2 \sigma}$ is compact we can find finitely many points $\left\{P_{1}, \ldots, P_{m}\right\} \subset K$ such that $K \subset \bigcup_{i=1}^{m} B_{\sigma^{\prime}-\sigma}\left(P_{i}\right)$ with $B_{2\left(\sigma^{\prime}-\sigma\right)}\left(P_{i}\right) \subset \bar{B}_{2 \sigma^{\prime}} \backslash B_{1 / 2 \sigma^{\prime}}$ for each $i=1, \ldots, m$. Then it suffices to show that $\left(\mathcal{P}_{k+1}^{\prime \prime}\right)$ holds in each ball $B_{i}:=B_{\sigma^{\prime}-\sigma}\left(P_{i}\right)$ assuming that $\left(\mathcal{P}_{k}^{\prime}\right),\left(\mathcal{P}_{k}^{\prime \prime}\right)$ and $\left(\mathcal{P}_{k+1}^{\prime}\right)$ hold in $B_{i}^{\prime}:=B_{2\left(\sigma^{\prime}-\sigma\right)}\left(P_{i}\right)$. For simplicity we shall drop the subscript $i$.

Taking (5.6) into account, we rewrite (5.7) as

$$
R^{-2} \Delta X_{R}=-\rho_{R}\left|\nabla w_{R}\right|^{2}+\rho_{R}\left(1+\rho_{R}\right) X_{R}
$$

Denoting by $D^{k}$ any $k$-th derivative, since $\left\{\rho_{R}\right\}_{R \geq R_{0}},\left\{X_{R}\right\}_{R \geq R_{0}},\left\{w_{R}\right\}_{R \geq R_{0}}$ and $\left\{\nabla w_{R}\right\}_{R \geq R_{0}}$ are bounded in $C^{k}\left(\overline{B^{\prime}}\right)$ by inductive assumption, differentiating 5.9$\}$ times leads to

$$
\left\|D^{k} X_{R}\right\|_{L^{\infty}\left(B^{\prime}\right)}+R^{-2}\left\|\Delta D^{k} X_{R}\right\|_{L^{\infty}\left(B^{\prime}\right)} \leq C,
$$

for some $C>0$ independent of $R \geq R_{0}$. Now we combine the above estimate with [5, Lemma A.1] in $B \subset B^{\prime \prime} \subset B^{\prime}$ where $B^{\prime \prime}:=B_{3\left(\sigma^{\prime}-\sigma\right) / 2}\left(P_{i}\right)$ to obtain

$$
R^{-1}\left\|D^{k+1} X_{R}\right\|_{L^{\infty}\left(B^{\prime \prime}\right)} \leq C
$$

for a constant $C>0$ independent of $R \geq R_{0}$. Finally we rewrite 5.9] as

$$
-R^{-2} \Delta X_{R}+2 X_{R}=3 R^{-2} X_{R}^{2}-R^{-4} X_{R}^{3}+\rho_{R}\left|\nabla w_{R}\right|^{2}=: \mathcal{T}_{R} .
$$

As we already proved that $D^{k+1} \rho_{R}$ is bounded in $B^{\prime \prime}$ independently of $R \geq R_{0}$ and that $\left(\mathcal{P}_{k}^{\prime \prime}\right),\left(\mathcal{P}_{k+1}^{\prime}\right)$ hold in $B^{\prime \prime}$, taking 5.10 into account we infer that $f_{R}:=D^{k+1} \mathcal{T}_{R}$ satisfies $\left\|f_{R}\right\|_{L^{\infty}\left(B^{\prime \prime}\right)} \leq C$ for a constant $C>0$ independent of $R \geq R_{0}$. Then differentiating 5.11) $k+1$ times we derive that $g_{R}:=D^{k+1} X_{R}$ satisfies

$$
\left\{\begin{array}{l}
-R^{-2} \Delta g_{R}+2 g_{R}=f_{R} \quad \text { in } B^{\prime \prime}, \\
\left\|g_{R}\right\|_{L^{\infty}\left(B^{\prime \prime}\right)} \leq C R, \\
\left\|f_{R}\right\|_{L^{\infty}\left(B^{\prime \prime}\right)} \leq C,
\end{array}\right.
$$


for some $C>0$ independent of $R \geq R_{0}$. Next we write $g_{R}=\varphi_{R}+\psi_{R}$ in $\overline{B^{\prime \prime}}$ where $\varphi_{R}$ and $\psi_{R}$ are the unique smooth solutions of

$$
\begin{cases}-R^{-2} \Delta \varphi_{R}+2 \varphi_{R}=0 & \text { in } B^{\prime \prime} \\ \varphi_{R}=g_{R} & \text { on } \partial B^{\prime \prime}\end{cases}
$$

and

$$
\begin{cases}-R^{-2} \Delta \psi_{R}+2 \psi_{R}=f_{R} & \text { in } B^{\prime \prime}, \\ \psi_{R}=0 & \text { on } \partial B^{\prime \prime} .\end{cases}
$$

Applying [5, Lemma 2] in $B \subset B^{\prime \prime}$ to 5.13, the comparison principle in $B^{\prime \prime}$ to 5.14, and the estimates in 5.12 we finally conclude

$$
\left\|D^{k+1} X_{R}\right\|_{L^{\infty}(B)}=\left\|g_{R}\right\|_{L^{\infty}(B)} \leq\left\|\varphi_{R}\right\|_{L^{\infty}(B)}+\left\|\psi_{R}\right\|_{L^{\infty}\left(B^{\prime \prime}\right)} \leq C
$$

for some $C>0$ independent of $R \geq R_{0}$, i.e., $\left(\mathcal{P}_{k+1}^{\prime \prime}\right)$ holds in $B$.

Remark 5.2. As a consequence of Proposition 5.2, Remark 5.1 and Proposition 4.1, if $u$ is an entire solution to (1.1) satisfying [5.1, then $\left\{u_{R \mid \mathbb{S}^{2}}\right\}_{R>0}$ is a compact subset of $C^{2}\left(\mathbb{S}^{2} ; \mathbb{R}^{3}\right)$ and the limit as $R_{n} \rightarrow+\infty$ of any convergent sequence $\left\{u_{R_{n} \mid \mathbb{S}^{2}}\right\}$ is a harmonic map $\omega \in C^{2}\left(\mathbb{S}^{2} ; \mathbb{S}^{2}\right)$ (more precisely, $\omega:=\phi_{\mid \mathbb{S}^{2}}$ where $\phi$ is given by Proposition 4.1. In addition, for $n$ large the topological degree of $u_{R_{n} \mid \mathbb{S}^{2}}$ is well defined and $\operatorname{deg} \omega=\operatorname{deg} u_{R_{n} \mid \mathbb{S}^{2}}=\operatorname{deg}_{\infty} u$.

In order to prove uniqueness of the asymptotic limit of a solution $u$ at infinity, we need to establish a decay estimate on the radial derivative of $u$. As will be clear below, such an estimate gives the existence of a limit for the scaled maps $u_{R}$ as $R \rightarrow+\infty$ in $L^{2}\left(\mathbb{S}^{2} ; \mathbb{R}^{3}\right)$. The a priori estimates in Proposition 5.2, as they yield compactness even in stronger topologies, will imply the convergence to an $\mathbb{S}^{2}$-valued harmonic map in $C^{k}\left(\mathbb{S}^{2} ; \mathbb{R}^{3}\right)$ for any $k \in \mathbb{N}$.

Proposition 5.3. Let $u$ be an entire solution of (1.1) satisfying (1.11). Then there exist $R_{0} \geq e$ and $C>0$ such that for any $R \geq R_{0}$,

$$
\int_{\{|x|>R\}} \frac{1}{|x|}\left|\frac{\partial u}{\partial r}\right|^{2} d x \leq C \frac{\log R}{R^{2}} .
$$

Proof. By (1.11) we can find $R_{0} \geq e$ such that $|u(x)| \geq 1 / 2$ whenever $|x| \geq R_{0}$. Then we perform the polar decomposition of $u$, i.e., for $|x| \geq R_{0}$ we write $u(x)=\rho(x) w(x)$ where $\rho(x)=|u(x)| \geq 1 / 2$ and $w(x) \in \mathbb{S}^{2}$. Due to (1.11) and 5.4), it is enough to prove 5.15 for $w$ since $\rho(x) \leq 1$ and $|\nabla \rho(x)|=\mathcal{O}\left(|x|^{-3}\right)$ as $|x| \rightarrow+\infty$. Taking 5.3 into account, we have $\nabla w(x)=\mathcal{O}\left(|x|^{-1}\right)$ and $\Delta w(x)=\mathcal{O}\left(|x|^{-2}\right)$ as $|x| \rightarrow+\infty$ so that equation (1.12) can be rewritten as

$$
\Delta w(x)+w(x)|\nabla w(x)|^{2}=G(x),
$$


where

$$
G(x)=\left(1-\rho^{2}(x)\right)\left(\Delta w(x)+w(x)|\nabla w(x)|^{2}\right)+\nabla w(x) \cdot \nabla\left(1-\rho^{2}(x)\right)=\mathcal{O}\left(|x|^{-4}\right)
$$

as $|x| \rightarrow+\infty$ thanks to (5.4). Next we multiply 5.16) by $\partial w / \partial r=(x /|x|) \cdot \nabla w$, and since $w$ and $\partial w / \partial r$ are orthogonal, we obtain

$$
0=(\Delta w-G(x)) \cdot \frac{\partial w}{\partial r}=\frac{1}{|x|}\left|\frac{\partial w}{\partial r}\right|^{2}+\operatorname{div} \Psi(x)-H(x),
$$

where

$$
\Psi(x)=\nabla w(x) \cdot \frac{\partial w}{\partial r}-\frac{1}{2}|\nabla w(x)|^{2} \frac{x}{|x|} \quad \text { and } \quad H(x)=G(x) \cdot \frac{\partial w}{\partial r}=\mathcal{O}\left(|x|^{-5}\right)
$$

as $|x| \rightarrow+\infty$ by (1.11), 5.3) and (5.4). Integrating (5.17) by parts in an annulus $A_{R^{\prime}, R}:=B_{R^{\prime} \backslash \overline{B_{R}}}$, with $R_{0} \leq R<R^{\prime}$ gives

$$
\begin{aligned}
\int_{A_{R^{\prime}, R}} \frac{1}{|x|}\left|\frac{\partial w}{\partial r}\right|^{2} d x-\frac{1}{2} \int_{\partial B_{R}}\left|\frac{\partial w}{\partial r}\right|^{2} d \mathcal{H}^{2} & =\frac{1}{2} \int_{\mathbb{S}^{2}}\left|\nabla_{T} w_{R^{\prime}}\right|^{2} d \mathcal{H}^{2}-\frac{1}{2} \int_{\mathbb{S}^{2}}\left|\nabla_{T} w_{R}\right|^{2} d \mathcal{H}^{2} \\
& +\frac{1}{2} \int_{\partial B_{R}^{\prime}}\left|\frac{\partial w}{\partial r}\right|^{2} d \mathcal{H}^{2}+\int_{A_{R^{\prime}, R}} H d x
\end{aligned}
$$

where $w_{R}$ and $w_{R^{\prime}}$ are defined as in Proposition 5.2 and $\nabla_{T}$ denotes the tangential gradient.

Since (1.11) obviously implies (1.8), the Monotonicity Formula 1.7$]$ yields

$$
\int_{\left\{|x|>R_{0}\right\}} \frac{1}{|x|}\left|\frac{\partial u}{\partial r}\right|^{2} d x<+\infty .
$$

Hence we can find a sequence $R_{n}^{\prime} \rightarrow+\infty$ such that

$$
\int_{\partial B_{R_{n}^{\prime}}}\left|\frac{\partial u}{\partial r}\right|^{2} d \mathcal{H}^{2} \stackrel{n \rightarrow+\infty}{\longrightarrow} 0
$$

In view of Remark 5.2 we can pass to a subsequence, still denoted by $\left\{R_{n}^{\prime}\right\}$, such that

$$
\left\|u_{R_{n}^{\prime} \mid \mathbb{S}^{2}}-\omega\right\|_{C^{2}\left(\mathbb{S}^{2} ; \mathbb{R}^{3}\right)} \stackrel{n \rightarrow+\infty}{\longrightarrow} 0
$$

for some smooth harmonic map $\omega: \mathbb{S}^{2} \rightarrow \mathbb{S}^{2}$ satisfying $\operatorname{deg} \omega=\operatorname{deg}_{\infty} u$. Taking 1.11 again into account, one can easily check that

$$
\begin{gathered}
\int_{|x|>R_{0}} \frac{1}{|x|}\left|\frac{\partial w}{\partial r}\right|^{2} d x<+\infty, \quad \int_{\partial B_{R_{n}^{\prime}}}\left|\frac{\partial w}{\partial r}\right|^{2} d \mathcal{H}^{2} \stackrel{n \rightarrow+\infty}{\longrightarrow} 0, \\
\int_{\mathbb{S}^{2}}\left|\nabla_{T} w_{R_{n}^{\prime}}\right|^{2} d \mathcal{H}^{2} \stackrel{n \rightarrow+\infty}{\longrightarrow} \int_{\mathbb{S}^{2}}\left|\nabla_{T} \omega\right|^{2} d \mathcal{H}^{2} .
\end{gathered}
$$


Choose $R^{\prime}=R_{n}^{\prime}$ in 5.18. Taking 5.21 into account and the integrability of $H$ at infinity, we can pass to the limit $R_{n}^{\prime} \rightarrow+\infty$ to obtain

$$
\begin{array}{rl}
\int_{\{|x|>R\}} \frac{1}{|x|}\left|\frac{\partial w}{\partial r}\right|^{2} & d x-\frac{1}{2} \int_{\partial B_{R}}\left|\frac{\partial w}{\partial r}\right|^{2} d \mathcal{H}^{2} \\
= & \frac{1}{2} \int_{\mathbb{S}^{2}}\left|\nabla_{T} \omega\right|^{2} d \mathcal{H}^{2}-\frac{1}{2} \int_{\mathbb{S}^{2}}\left|\nabla_{T} w_{R}\right|^{2} d \mathcal{H}^{2}+\int_{\{|x|>R\}} H d x
\end{array}
$$

for each $R \geq R_{0}$. Then observe that $\operatorname{deg} w_{R \mid \mathbb{S}^{2}}=\operatorname{deg} \omega$ for each $R \geq R_{0}$ by Remark 5.2. On the other hand, $\omega: \mathbb{S}^{2} \rightarrow \mathbb{S}^{2}$ is a harmonic map so that $\omega$ is energy minimizing in its own homotopy class. Therefore,

$$
\int_{\mathbb{S}^{2}}\left|\nabla_{T} \omega\right|^{2} d \mathcal{H}^{2} \leq \int_{\mathbb{S}^{2}}\left|\nabla_{T} w_{R}\right|^{2} d \mathcal{H}^{2}
$$

Multiplying (5.22) by $2 R$ and using (5.23), we derive

$$
\frac{d}{d R}\left(R^{2} \int_{\{|x|>R\}} \frac{1}{|x|}\left|\frac{\partial w}{\partial r}\right|^{2} d x\right) \leq 2 R \int_{\{|x|>R\}} H d x
$$

for every $R>R_{0}$. Integrating the above inequality between $R_{0}$ and $R>R_{0}$, using $H(x)=\mathcal{O}\left(|x|^{-5}\right)$ and (5.21), we finally obtain

$$
R^{2} \int_{\{|x|>R\}} \frac{1}{|x|}\left|\frac{\partial w}{\partial r}\right|^{2} d x \leq R_{0}^{2} \int_{\left\{|x|>R_{0}\right\}} \frac{1}{|x|}\left|\frac{\partial w}{\partial r}\right|^{2} d x+C \int_{R_{0}}^{R} \frac{1}{r} d r \leq C(\log R+1),
$$

and the proof is complete.

Now we are in a position to prove the asymptotic symmetry of entire solutions of (1.1).

Proof of Theorem 1.3. Since $u$ satisfies (1.8) and $\left\{u_{R}\right\}_{R>0}$ is relatively compact in $H_{\text {loc }}^{1}\left(\mathbb{R}^{3} ; \mathbb{R}^{3}\right.$ ), we can apply Propositions 5.1 and 5.2 to obtain 1.11 . Next we fix $R_{0}$ as in Proposition 5.3 and we estimate for $R_{0} \leq \tau_{1} \leq \tau_{2} \leq 2 \tau_{1}$,

$$
\begin{aligned}
\left|u_{\tau_{1}}(\sigma)-u_{\tau_{2}}(\sigma)\right|^{2} & \leq\left(\tau_{2}-\tau_{1}\right) \int_{\tau_{1}}^{\tau_{2}}\left|\frac{\partial u}{\partial r}(r \sigma)\right|^{2} d r \\
& \leq \int_{\tau_{1}}^{\tau_{2}}\left|\frac{\partial u}{\partial r}(r \sigma)\right|^{2} r d r \quad \text { for every } \sigma \in \mathbb{S}^{2}
\end{aligned}
$$

Integrating the previous inequality with respect to $\sigma$, we infer from 5.15 that

$$
\begin{aligned}
\int_{\mathbb{S}^{2}}\left|u_{\tau_{1}}-u_{\tau_{2}}\right|^{2} d \mathcal{H}^{2} & \leq \int_{\left\{\tau_{1} \leq|x| \leq \tau_{2}\right\}} \frac{1}{|x|}\left|\frac{\partial u}{\partial r}\right|^{2} d x \\
& \leq C \frac{\log \tau_{1}}{\tau_{1}^{2}} \quad \text { for every } R_{0} \leq \tau_{1} \leq \tau_{2} \leq 2 \tau_{1},
\end{aligned}
$$

where the constant $C$ only depends on $R_{0}$. 
Next we consider $R_{0} \leq R<R^{\prime}$ arbitrary. Define $k \in \mathbb{N}$ to be the largest integer satisfying $2^{k} R \leq R^{\prime}$, and set $\tau_{j}:=2^{j} R$ for $j=0, \ldots, k$ and $\tau_{k+1}:=R^{\prime}$. Using (5.24) together with the triangle inequality, we estimate

$$
\begin{aligned}
\left\|u_{R}-u_{R^{\prime}}\right\|_{L^{2}\left(\mathbb{S}^{2}\right)} & \leq \sum_{j=0}^{k}\left\|u_{\tau_{j}}-u_{\tau_{j+1}}\right\|_{L^{2}\left(\mathbb{S}^{2}\right)} \leq C \sum_{j=0}^{k} \frac{\sqrt{\log \tau_{j}}}{\tau_{j}} \\
& \leq \frac{C}{R} \sum_{j=0}^{\infty} \frac{\sqrt{j \log 2+\log R}}{2^{j}} \leq C \frac{\sqrt{\log R}}{R}
\end{aligned}
$$

for a constant $C$ which only depends on $R_{0}$. Obviously this estimate yields the uniqueness of the limit $\omega:=\lim _{R \rightarrow+\infty} u_{R \mid \mathbb{S}^{2}}$ in the $L^{2}$-topology. In view of Remark 5.2 the convergence also holds in the $C^{2}$-topology and $\omega: \mathbb{S}^{2} \rightarrow \mathbb{S}^{2}$ is a smooth harmonic map satisfying $\operatorname{deg} \omega=\operatorname{deg}_{\infty} u$. So claim (i) in the theorem is proved. Then from (i), (1.11) and Proposition 4.1 we deduce that $u_{R} \rightarrow u_{\infty}$ strongly in $H_{\text {loc }}^{1}\left(\mathbb{R}^{3} ; \mathbb{R}^{3}\right)$ as $R \rightarrow+\infty$ with $u_{\infty}(x)=\omega(x /|x|)$, proving claim (ii).

Moreover claim (ii) in Proposition 4.1 yields

$$
\int_{S^{2}} x\left|\nabla_{T} \omega\right| d \mathcal{H}^{2}=0
$$

As a consequence, if $\operatorname{deg}_{\infty} u= \pm 1=\operatorname{deg} \omega$ the balancing condition above gives $\omega(x)=T x$ for some $T \in O(3)$ by [8, proof of Theorem 7.3].

\section{Proof of Theorem 1.4}

Proof of (i) $\Rightarrow$ (ii). This is just Theorem 1.2

Proof of (ii) $\Rightarrow$ (iii). First we claim that the scaled maps $\left\{u_{R}\right\}_{R>0}$ given by (1.9) are compact in $H_{\text {loc }}^{1}\left(\mathbb{R}^{3} ; \mathbb{R}^{3}\right)$. Indeed, by (ii) we can apply Proposition 4.1 to infer that for any weakly convergent sequence $\left\{u_{R_{n}}\right\}$ as $R_{n} \rightarrow+\infty$ we have

$$
\int_{B_{1}} \frac{1}{2}|\nabla \phi|^{2} d x+v\left(B_{1}\right)=4 \pi,
$$

where $\phi$ is the weak limit of $\left\{u_{R_{n}}\right\}$ and $v$ is the defect measure as in Proposition 4.1. If $v \neq 0$ the above equality together with the structure of $v$ yields $\phi \equiv$ const and $l=k_{1}=1$, which contraddicts the balancing condition in Proposition 4.1 (ii). Hence $v \equiv 0$ and $\left\{u_{R_{n}}\right\}$ is strongly convergent in $H_{\text {loc }}^{1}\left(\mathbb{R}^{3} ; \mathbb{R}^{3}\right)$.

Now we can apply Theorem 1.3 to get 1.11 which obviously implies $|u(x)|=1+$ $\mathcal{O}\left(|x|^{-2}\right)$ as $|x| \rightarrow+\infty$. Moreover $u_{R} \rightarrow u_{\infty}$ strongly in $H_{\text {loc }}^{1}\left(\mathbb{R}^{3} ; \mathbb{R}^{3}\right)$ as $R \rightarrow+\infty$ where $u_{\infty}(x)=\omega(x /|x|)$ for some smooth harmonic map $\omega: \mathbb{S}^{2} \rightarrow \mathbb{S}^{2}$ satisfying $\operatorname{deg} \omega=\operatorname{deg}_{\infty} u$. Therefore,

$$
4 \pi|\operatorname{deg} \omega|=\int_{B_{1}} \frac{1}{2}\left|\nabla u_{\infty}\right|^{2} d x=\lim _{R \rightarrow+\infty} E_{R}\left(u_{R}, B_{1}\right)=\lim _{R \rightarrow+\infty} \frac{1}{R} E\left(u, B_{R}\right)=4 \pi,
$$

so that $\operatorname{deg} \omega=\operatorname{deg}_{\infty} u= \pm 1$. 
Proof of (iii) $\Rightarrow$ (iv). From Remark 5.1 we deduce that $u$ satisfies $(1.8)$ and the scaled maps $\left\{u_{R}\right\}_{R>0}$ are compact in $H_{\text {loc }}^{1}\left(\mathbb{R}^{3} ; \mathbb{R}^{3}\right)$. As a consequence we can apply Theorem 1.3 to obtain estimate (1.11). In addition, up to an orthogonal transformation we may assume $\operatorname{deg}_{\infty} u=1$ and $\left\|u_{R}-\operatorname{Id}\right\|_{C^{2}\left(\mathbb{S}^{2} ; \mathbb{R}^{3}\right)} \rightarrow 0$ as $R \rightarrow+\infty$. By degree theory we have $u^{-1}(\{0\}) \neq \emptyset$ and up to a translation, we may also assume that $u(0)=0$.

Now we are in a position to apply the division trick of [24] (see also [25] for another application). Let $f \in C^{2}([0, \infty))$ be given by Lemma 2.1 and define

$$
v(x):=\frac{u(x)}{f(|x|)} .
$$

Clearly $v \in C^{2}\left(\mathbb{R}^{3} \backslash\{0\} ; \mathbb{R}^{3}\right)$, and it is straightforward to check that as $|x| \rightarrow 0$,

$v(x)=B \frac{x}{|x|}+o(1), \quad \nabla v(x)=\nabla\left(B \frac{x}{|x|}\right)+o\left(|x|^{-1}\right), \quad$ where $B:=\frac{\nabla u(0)}{f^{\prime}(0)}$.

On the other hand, using Lemma 2.1 and the behavior of $u$ at infinity, one can check that as $|x| \rightarrow+\infty$,

$$
v(x)=\frac{x}{|x|}+o(1), \quad \nabla v(x)=\nabla\left(\frac{x}{|x|}\right)+o\left(|x|^{-1}\right) .
$$

Since $u$ solves (1.1) and $f$ solves 2.1], simple computations lead to

$$
\Delta v+f^{2} v\left(1-|v|^{2}\right)=-2 \frac{f^{\prime}}{f} \frac{x}{|x|} \cdot \nabla v-\frac{2}{|x|^{2}} v .
$$

Multiplying this equation by $\frac{\partial v}{\partial r}=\frac{x}{|x|} \cdot \nabla v$ yields

$$
0 \leq\left|\frac{\partial v}{\partial r}\right|^{2}\left(\frac{1}{|x|}+2 \frac{f^{\prime}}{f}\right)+\left(\frac{\left(1-|v|^{2}\right)^{2}}{4}\right)\left(2 f f^{\prime}+\frac{2}{|x|}\right)=\operatorname{div} \Phi(x),
$$

where

$$
\Phi(x):=\left(\frac{1}{2}|\nabla v|^{2} \frac{x}{|x|}\right)-\left(\nabla v \cdot \frac{\partial v}{\partial r}\right)+\left(\frac{x}{|x|} f^{2} \frac{\left(1-|v|^{2}\right)^{2}}{4}\right)+\left(\frac{x}{|x|^{3}}\left(1-|v|^{2}\right)\right) .
$$

Now we claim that

$$
\int_{B_{R} \backslash B_{\delta}} \operatorname{div} \Phi d x=\int_{\{|x|=R\}} \Phi(x) \cdot \frac{x}{|x|} d \mathcal{H}^{2}-\int_{\{|x|=\delta\}} \Phi(x) \cdot \frac{x}{|x|} d \mathcal{H}^{2} \rightarrow 0
$$

as $R \rightarrow+\infty$ and $\delta \rightarrow 0$. Assume that the claim is proved. Then from 6.3 we infer that $|v| \equiv 1$ and $\partial v / \partial r \equiv 0$. As a consequence, in view of $[6.2\rangle$ we derive that $|u(x)| \equiv f(|x|)$ and $v(x) \equiv x /|x|$, which concludes the proof.

In order to prove 6.4 we first observe that as $|x| \rightarrow+\infty$,

$$
|\nabla v|^{2}=\frac{2}{|x|^{2}}+o\left(|x|^{-2}\right), \quad \frac{\partial v}{\partial r}=o\left(|x|^{-1}\right), \quad 1-|v|^{2}=\mathcal{O}\left(|x|^{-2}\right),
$$


thanks to 6.2 and (iii). Therefore,

$\int_{\{|x|=R\}} \Phi(x) \cdot \frac{x}{|x|} d \mathcal{H}^{2}=\int_{\{|x|=R\}}\left(\frac{1}{|x|^{2}}+o\left(|x|^{-2}\right)\right) d \mathcal{H}^{2}=4 \pi+o(1) \quad$ as $R \rightarrow+\infty$.

Next, using 6.1, we estimate as $|x| \rightarrow 0$,

$|\nabla v|^{2}=\left|\nabla\left(B \frac{x}{|x|}\right)\right|^{2}+o\left(|x|^{-2}\right), \quad \frac{\partial v}{\partial r}=o\left(|x|^{-1}\right), \quad 1-|v|^{2}=\frac{|x|^{2}-|B x|^{2}}{|x|^{2}}+o(1)$.

Consequently,

$$
\begin{gathered}
\int_{\{|x|=\delta\}} \Phi(x) \cdot \frac{x}{|x|} d \mathcal{H}^{2}=\int_{\{|x|=\delta\}}\left(\frac{1}{2}\left|\nabla\left(B \frac{x}{|x|}\right)\right|^{2}+\frac{|x|^{2}-|B x|^{2}}{|x|^{4}}+o\left(|x|^{-2}\right)\right) d \mathcal{H}^{2} \\
=\int_{\{|x|=1\}}\left(\frac{1}{2}\left|\nabla\left(B \frac{x}{|x|}\right)\right|^{2}-\frac{|B x|^{2}}{|x|^{4}}\right) d \mathcal{H}^{2}+4 \pi+o(1) \quad \text { as } \delta \rightarrow 0 .
\end{gathered}
$$

Since a direct computation gives

$$
\int_{\{|x|=1\}}\left(\frac{1}{2}\left|\nabla\left(A \frac{x}{|x|}\right)\right|^{2}-\frac{|A x|^{2}}{|x|^{4}}\right) d \mathcal{H}^{2}=0
$$

for any constant matrix $A \in \mathbb{R}^{3 \times 3}$, claim 6.4 follows by combining 6.5 and 6.6 .

Proof of (iv) $\Rightarrow$ (i). Let $u$ be a nonconstant local minimizer as given by Theorem 1.1 Since $R^{-1} E\left(u, B_{R}\right) \rightarrow 4 \pi$ as $R \rightarrow+\infty$ and $u(0)=0$, and as we already proved (ii) $\Rightarrow$ (iii) $\Rightarrow$ (iv), we conclude that up to a rotation, $u(x)=U(x)$ as given by (1.4). Hence $U$ is a nonconstant local minimizer of the energy, which is still the case when composing with translations and orthogonal transformations.

Acknowledgments. The authors would like to thank Fabrice Bethuel, Alberto Farina and Giovanni Leoni for useful discussions. This work was initiated while A.P. was visiting the Carnegie Mellon University. He would like to thank Irene Fonseca for the kind invitation and the warm hospitality. V.M. was partially supported by the Center for Nonlinear Analysis (CNA) under the National Science Foundation Grant No. 0405343.

\section{References}

[1] Akopian, V., Farina, A.: Sur les solutions radiales de l'équation $-\Delta u=u\left(1-|u|^{2}\right)$ dans $\mathbb{R}^{N}(N \geq 3)$. C. R. Acad. Sci. Paris Sér. I Math. 325, 601-604 (1997) Zbl 0890.35044 MR 1473831

[2] Almgren, F. J., Lieb, E. H.: Singularities of energy minimizing maps from the ball to the sphere: examples, counterexamples, and bounds. Ann. of Math. (2) 128, 483-530 (1988) Zbl 0673.58013 MR 0970609

[3] Ambrosio, L., Cabré, X.: Entire solutions of semilinear elliptic equations in $\mathbb{R}^{3}$ and a conjecture of De Giorgi. J. Amer. Math. Soc. 13, 725-739 (2000) Zbl 0968.35041 MR 1775735 
[4] Ambrosio, L., Fusco, N., Pallara, D.: Functions of Bounded Variation and Free Discontinuity Problems. Oxford Math. Monogr., Oxford Univ. Press, New York (2000) Zbl 0957.49001 MR 1857292

[5] Bethuel, F., Brezis, H., Hélein, F.: Asymptotics for the minimization of a GinzburgLandau functional. Calc. Var. Partial Differential Equations 1, 123-148 Zbl 0834.35014 MR 1261720

[6] Bethuel, F., Brezis, H., Hélein, F.: Ginzburg-Landau Vortices. Progr. Nonlinear Differential Equations Appl. 13, Birkhäuser, Boston (1994) Zbl 0802.35142 MR 1269538

[7] Brezis, H.: Symmetry in nonlinear PDE's. In: Differential Equations: La Pietra 1996 (Florence), Proc. Sympos. Pure Math. 65, Amer. Math. Soc., Providence, 1-12 (1999) Zbl 0927.35038 MR 1662746

[8] Brezis, H., Coron, J. M., Lieb, E. H.: Harmonic maps with defects. Comm. Math. Phys. 107 (1986), 649-705. Zbl 0608.58016 MR 0868739

[9] Chen, Y.: Dirichlet problems for heat flows of harmonic maps in higher dimensions. Math. Z. 208, 557-565 (1991) Zbl 0727.58012 MR 1136475

[10] Chen, Y., Lin, F. H.: Evolution of harmonic maps with Dirichlet boundary conditions. Comm. Anal. Geom. 1, 327-346 (1993) Zbl 0845.35049 MR 1266472

[11] Chen, Y., Struwe, M.: Existence and partial regularity results for the heat flow for harmonic maps. Math. Z. 201, 83-103 (1989) Zbl 0652.58024 MR 0990191

[12] Farina, A.: Finite-energy solutions, quantization effects and Liouville-type results for a variant of the Ginzburg-Landau system in $\mathbb{R}^{k}$. Differential Integral Equations 11, 875-893 (1998) Zbl 1074.35504 MR 1659256

[13] Farina, A., Guedda, M.: Qualitative study of radial solutions of the Ginzburg-Landau system in $\mathbb{R}^{N}(N \geq 3)$. Appl. Math. Lett. 13, 59-64 (2000) Zbl 0954.35059 MR 1777804

[14] Gulliver, R., White, B.: The rate of convergence of a harmonic map at a singular point. Math. Ann. 283, 539-549 (1989) Zbl 0645.58018 MR 0990588

[15] Gustafson, S.: Symmetric solutions of the Ginzburg-Landau equation in all dimensions. Int. Math. Res. Notices 1997, no. 16, 807-816 Zbl 0883.35041 MR 1472346

[16] Hagan, P. S.: Spiral waves in reaction-diffusion systems. SIAM J. Appl. Math. 42, 762-786 (1982) Zbl 0507.35007 MR 0665385

[17] Hervé, R. M., Hervé, M.: Étude qualitative des solutions réelles d'une équation différentielle liée à l'équation de Ginzburg-Landau. Ann. Inst. H. Poincaré 11, 427-440 (1994) Zbl 0836.34090 MR 1287240

[18] Jendoubi, M. A.: A simple unified approach to some convergence theorems of L. Simon. J. Funct. Anal. 153, 187-202 (1998) Zbl 0895.35012 MR 1609269

[19] Kawohl, B.: Symmetrization - or how to prove symmetry of solutions to a PDE. In: Partial Differential Equations (Praha, 1998), Chapman \& Hall/CRC Res. Notes Math. 406, Chapman \& Hall and CRC, Boca Raton, FL, 214-229 (2000) Zbl 0933.35013 MR 1713887

[20] Lin, F. H., Rivière, T.: Energy quantization for harmonic maps. Duke Math. J. 111, 177-193 (2002) Zbl 1014.58008 MR 1876445

[21] Lin, F. H., Wang, C. Y.: Harmonic and quasi-harmonic spheres. Comm. Anal. Geom. 7, 397429 (1999) Zbl 0934.58018 MR 1685578

[22] Lin, F. H., Wang, C. Y.: Harmonic and quasi-harmonic spheres. II. Comm. Anal. Geom. 10, 341-375 (2002) Zbl 1042.58005 MR 1900755

[23] Mironescu, P.: On the stability of radial solutions of the Ginzburg-Landau equation. J. Funct. Anal. 130, 334-344 (1995) Zbl 0839.35011 MR 1335384

[24] Mironescu, P.: Les minimiseurs locaux pour l'équation de Ginzburg-Landau sont à symétrie radiale. C. R. Acad. Sci. Paris Sér. I Math. 323, 593-598 (1996) Zbl 0858.35038 MR 1411048 
[25] Rivière, T.: Towards Jaffe and Taubes conjectures in the strongly repulsive limit. Manuscripta Math. 108, 217-273 (2002) Zbl 1130.35340 MR 1918588

[26] Sandier, E.: Locally minimizing solutions of $-\Delta u=u\left(1-|u|^{2}\right)$ in $\mathbb{R}^{2}$. Proc. Roy. Soc. Edinburgh Sect. A 128, 349-358 (1998) Zbl 0905.35018 MR 1621347

[27] Schoen, R.: Analytic aspects of the harmonic map problem. In: Seminar on Nonlinear Partial Differential Equations (Berkeley, 1983). Math. Sci. Res. Inst. Publ. 2, Springer, New York, 321-358 (1984) Zbl 0551.58011 MR 0765241

[28] Shafrir, I.: Remarks on solutions of $-\Delta u=\left(1-|u|^{2}\right) u$ in $\mathbb{R}^{2}$. C. R. Acad. Sci. Paris Sér. I 318, 327-331 (1994) Zbl 0806.35030 MR 1267609

[29] Simon, L.: Asymptotics for a class of nonlinear evolution equations, with applications to geometric problems. Ann. of Math. (2) 118, 525-571 (1983) Zbl 0549.35071 MR 0727703

[30] Simon, L.: Theorems on Regularity and Singularity of Energy Minimizing Maps. Lectures in Math. ETH Zürich, Birkhäuser, Basel (1996) Zbl 0864.58015 MR 1463752 